\title{
Asymptotic behavior of cutoff effects in Yang-Mills theory and in Wilson's lattice QCD
}

\author{
Nikolai Husung $^{2, \mathrm{a}}$, Peter Marquard ${ }^{3, \mathrm{~b}}$, Rainer Sommer ${ }^{1,2, \mathrm{c}}$ \\ ${ }^{1}$ Institut für Physik, Humboldt-Universität zu Berlin, Newtonstr. 15, 12489 Berlin, Germany \\ 2 John von Neumann Institute for Computing (NIC), DESY, Platanenallee 6, 15738 Zeuthen, Germany \\ ${ }^{3}$ DESY, Platanenallee 6, 15738 Zeuthen, Germany
}

Received: 18 December 2019 / Accepted: 26 January 2020 / Published online: 3 March 2020

(C) The Author(s) 2020

\begin{abstract}
Discretization effects of lattice QCD are described by Symanzik's effective theory when the lattice spacing, $a$, is small. Asymptotic freedom predicts that the leading asymptotic behavior is $\sim a^{n_{\min }}\left[\bar{g}^{2}\left(a^{-1}\right)\right]^{\hat{\gamma}_{1}} \sim a^{n_{\min }}\left[\frac{1}{-\log (a \Lambda)}\right]^{\hat{\gamma}_{1}}$. For spectral quantities, $n_{\min }=d$ is given in terms of the (lowest) canonical dimension, $d+4$, of the operators in the local effective Lagrangian and $\hat{\gamma}_{1}$ is proportional to the leading eigenvalue of their one-loop anomalous dimension matrix $\gamma^{(0)}$. We determine $\gamma^{(0)}$ for Yang-Mills theory $\left(n_{\min }=2\right)$ and discuss consequences in general and for perturbatively improved short distance observables. With the help of results from the literature, we also discuss the $n_{\min }=1$ case of Wilson fermions with perturbative $\mathrm{O}(a)$ improvement and the discretization effects specific to the flavor currents. In all cases known so far, the discretization effects are found to vanish faster than the naive $\sim a^{n_{\text {min }}}$ behavior with rather small logarithmic corrections - in contrast to the two-dimensional $\mathrm{O}(3)$ sigma model.
\end{abstract}

\section{Contents}

1 Introduction ................. 1

Scope . . . . . . . . . . . . . 2

2 Symanzik effective theory and logarithmic corrections to $a^{n}$ behavior . . . . . . . . . . . 2

3 One-loop computation of the anomalous dimension matrix ............... . 5 5

4 Matching to lattice actions . . . . . . . . . . 7

5 Examples for the asymptotic behavior . . . . . . . 8

5.1 Generic form for spectral quantities . . . . . 8

5.2 Short distance observables . . . . . . . . 8

\footnotetext{
a e-mail: nikolai.husung@desy.de

b e-mail: peter.marquard@desy.de

c e-mail: rainer.sommer@desy.de (corresponding author)
}

Improved observables . . . . . . . . . . . . . 9

6 Schrödinger functional . . . . . . . . . . . 10

7 Wilson-QCD ............... 12

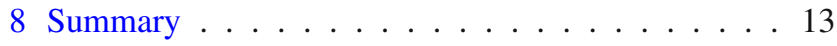

References................. 14

\section{Introduction}

Lattice regularizations provide a definition of quantum field theories beyond perturbation theory. Evaluating the associated path integral by Monte Carlo also represents a nonperturbative calculational method to derive predictions from the theory. One of the systematic effects that have to be taken into account is the dependence of results on the lattice spacing $a$ (we assume a hyper-cubic lattice throughout) or in other words the size of discretization errors,

$\Delta_{\mathcal{P}}(a)=\mathcal{P}(a)-\mathcal{P}(0)$,

associated with a dimensionless observable $\mathcal{P}$ of the theory. As a start, one may consider the classical field theory. One then has smooth fields, and the lattice-Lagrangian can be simply Taylor expanded. It is the continuum one up to terms suppressed by powers of $a$.

One may therefore think that also in the full, quantized, theory the small- $a$ behavior of the discretization errors is $\Delta_{\mathcal{P}}(a)=p_{1} a^{n_{\min }}+p_{2} a^{n_{\min }+1}+\cdots$ with the integer $n_{\text {min }}$ given by the first non-zero power in the classical Taylor expansion of the Lagrangian. However, the divergences of quantum field theories spoil this behavior.

Still, precise statements can be made about the small- $a$ expansion, based on Symanzik's effective theory (SymEFT) [1-4], see also [5, p. 39ff.]. It describes the small- $a$ behavior by an effective field theory with a local Lagrangian

$$
\mathscr{L}_{\mathrm{eff}}(x)=\mathscr{L}+a \delta \mathscr{L}^{(1)}(x)+a^{2} \delta \mathscr{L}^{(2)}(x)+\cdots
$$


The effective theory can be thought of as a continuum effective theory, regularized e.g. by dimensional regularization. The first term is the continuum Lagrangian $\mathscr{L}$ of the fundamental field theory and $\delta \mathscr{L}^{(d)}(x)$ are local Lagrangians of higher mass dimension. The leading term in Eq. (1.1) is then given by the one ${ }^{1}$ with the lowest mass dimension in Eq. (1.2), i.e. $\delta \mathscr{L}^{(1)}(x)$, unless it vanishes. The corrections $\delta \mathscr{L}^{(d)}(x)$ can be written as a linear combination of basis operators $\mathcal{B}_{i}(x)$ with the appropriate canonical mass dimensions. Renormalization of the effective theory introduces anomalous dimensions for the operators $\mathcal{B}_{i}$. It may therefore modify the small- $a$ expansion to $\Delta_{\mathcal{P}}(a)=p_{1} a^{n_{\min }+\eta}+\cdots$ with, in general, non-integer $\eta$. The (leading) anomalous dimension $\eta$ is in general a non-perturbative quantity, but it may sometimes be estimated by perturbation theory in the $\epsilon$-expansion, see [6].

We now turn to asymptotically free theories such as QCD. There, small $a$ means weak coupling at the scale of the lattice cutoff and the anomalous dimension can (1) be computed in perturbation theory and (2) it leads to a modification of $a^{n}$ by $\operatorname{logs}[1,2,7,8]$,

$$
\Delta_{\mathcal{P}}(a)=p_{1}[-\log (a \Lambda)]^{-\hat{\gamma}} a^{n_{\min }}+\cdots
$$

and not by fractional powers. The intrinsic scale of the theory, $\Lambda$, is a renormalization group invariant and the exponent $\hat{\gamma}$ is proportional to a one-loop anomalous dimension. Since the work of [9], continuum extrapolations are routinely performed in order to obtain quantitative numbers for continuum field theory observables. They have been carried out with just powers ${ }^{2}$ of $a$, thus implicitly assuming that $\hat{\gamma}$ is small. Of course this can not really be taken for granted until $\hat{\gamma}$ is known from a computation. Here we start to fill this gap.

Note that the logarithmic corrections in Eq. (1.3) can be very relevant. An explicit example is provided by the seminal work of Balog, Niedermayer and Weisz [7,8]. It concerns the 2-d $\mathrm{O}(3)$ sigma model where the leading term is $\hat{\gamma}=-3$ and the logarithmic corrections change the naive $a^{2}$ behavior to a shape which numerically looks like $a$ in a broad range of $a \Lambda[7,8]$. This numerical behavior led to quite some concern [10] and the computation of the logarithmic corrections by Balog, Niedermayer and Weisz were essential to confirm that the SymEFT description holds and put continuum extrapolations on a solid ground. In lattice QCD, knowledge of the leading power of the logarithms (and partially awareness of the issue) are still missing; in particular it is important to have a confirmation that $\hat{\gamma}$ is small as is usually assumed. Let us cite Peter Weisz [5]:

\footnotetext{
1 We will be more precise below.

${ }^{2}$ Sometimes an additional power of $\bar{g}^{2}\left(a^{-1}\right) \sim[-\log (a \Lambda)]^{-1}$ has been used when a tree-level improved action is used. Here $\bar{g}^{2}$ is the running coupling in some scheme.
}

The program should be carried out for lattice actions used for large scale simulations of $Q C D$, when technically possible, in order to check if potentially large logarithmic corrections to lattice artifacts predicted by perturbative analysis appear. Ten years later, as a first step, we do carry out the program in the pure Yang-Mills (YM) theory as well as in Wilson's lattice QCD without non-perturbative $\mathrm{O}(a)$ improvement. The latter case is rather simple and basically given by results in the literature. We will therefore discuss only the YM theory in detail and just mention the difference and results in Wilson's QCD in Sect. 7.

\section{Scope}

In addition to the discretization effects due to the terms $\delta \mathscr{L}^{(d)}$ in the effective Lagrangian, correlation functions of local fields $\Phi(x)$ also get $a$-effects from corrections to the fields $\Phi(x)$ represented in the SymEFT [11,12]. Apart from mostly restricting ourselves to the YM theory, we also do not discuss these additional discretization effects. They are absent in quantities which are independent of details of the local fields. We call those spectral quantities, since the spectrum of the Hamiltonian is the important application. In the YM theory, correlation functions themselves have so far not played a relevant role, apart from one notable exception. The exception is the new sector of Gradient flow observables $[13,14]$. We leave its treatment to future work.

\section{Symanzik effective theory and logarithmic corrections to $a^{n}$ behavior}

We consider YM theory in 4 dimensions defined by the action

$$
\begin{aligned}
& S_{\text {lat }}=\frac{2}{g_{0}^{2}} \sum_{x, \mu>v=0}^{3} p(x, \mu, v), \\
& \quad p(x, \mu, v)=\operatorname{Re} \operatorname{tr}(1- \\
& \left.\quad U(x, \mu) U(x+a \hat{\mu}, v) U^{-1}(x+a \hat{v} \mu) U^{-1}(x, v)\right)
\end{aligned}
$$

in terms of the link variables $U(x, \mu) \in \mathrm{SU}(\mathrm{N})$, connecting $x+a \hat{\mu}$ and $x$. We assume a lattice with periodic boundary conditions in space and infinite (or arbitrarily large) time extent. $^{3}$

As an example of a simple observable, $\mathcal{P}$, take a ratio of glue-ball masses, which may be defined as ( $\partial_{\mu}^{\text {lat }} f(x)=$ $[f(x+a \hat{\mu})-f(x)] / a$ and $\left.x=\left(x_{0}, \mathbf{x}\right)\right)$

\footnotetext{
3 In practice, finite lattices are of course needed for the Monte Carlo evaluation. The appropriate modifications of equations such as Eq. (2.2) are standard.
} 
$\mathcal{P}=m_{i}^{\mathrm{h}} / m_{j}^{\mathrm{h}}, \quad m_{i}^{\mathrm{h}}=-\lim _{x_{0} \rightarrow \infty} \partial_{0}^{\mathrm{lat}} \log \left(a^{3} \sum_{\mathbf{x}} C_{i}(x)\right)$,

in terms of a two-point function

$C_{i}(x-y)=\left\langle\Phi_{i}(x) \Phi_{i}(y)\right\rangle_{\text {lat }}^{\text {con }}$.

The gauge invariant fields $\Phi_{i}(x)$ are formed out of small (with a maximal extent $r_{\mathrm{w}}$ with $r_{\mathrm{w}} / a$ fixed) spatial Wilson loops, combined in such a way as to have a definite transformation under the lattice cubic group. A very simple example is the scalar field $\Phi_{1}(x)=Z_{F^{2}} \sum_{k, l \in\{1,2,3\}} p(x, k, l)$. For simplicity we assume in the following that the renormalization factors, such as $Z_{F^{2}}$, are determined such that they do not introduce any cutoff effects. In perturbation theory minimal (lattice) subtraction has this property. Expectation values are defined by the lattice path integral

$\langle F(U)\rangle_{\text {lat }}=\frac{1}{\mathcal{Z}} \int \prod_{x, \mu} \mathrm{d} U(x, \mu) \mathrm{e}^{-S_{\text {lat }}(U)} F(U)$,

where $\mathcal{Z}$ normalizes such that $\langle 1\rangle_{\text {lat }}=1, F(U)$ stands for a function of any number of link variables $U(x, \mu)$ and $\mathrm{d} U(x, \mu)$ is the invariant Haar measure. The label "con" stands for connected correlation functions, namely the subtraction of $\left[\left\langle\Phi_{i}(x)\right\rangle_{\text {lat }}\right]^{2}$ in Eq. (2.3)

Note that while $C_{i}(x)$ depend on the details of the definition of $\Phi_{i}(x)$, the masses $m_{i}^{\mathrm{h}}$ only depend on the quantum numbers of the field $\Phi_{i}(x)$. Masses or more generally energies are spectral quantities.

SymEFT gives the small- $a$ expansion of correlation functions such as $C_{i}(x)$ in the form of a continuum effective field theory. The central statement is

$C(x)=C^{\mathrm{cont}}(x)+a^{n_{\min }} \delta C(x)+\mathrm{O}\left(a^{n_{\min }+1}\right)$

and the expansion on the r.h.s. can be obtained from the effective continuum field theory with effective Lagrangian Eq. (1.2) supplemented by correction terms which are due to correction terms of the fields $[11,12]$

$\Phi_{\mathrm{eff}}(x)=\Phi(x)+a \delta \Phi^{(1)}(x)+a^{2} \delta \Phi^{(2)}(x)+\cdots$.

Let us mention right away that $n_{\min }=2$ in the considered YM theory.

For precise statements we need to specify

1. the rules of the EFT, i.e. how precisely are $\delta C(x)$ defined in terms of $\delta \mathscr{L}^{(d)}(x), \delta \Phi^{(d)}(x)$,

2. which local operators contribute to $\delta \mathscr{L}^{(d)}(x), \delta \Phi^{(d)}(x)$,

3 . how are the parameters of the EFT determined, in other words how are the coefficients of those operators contributing to $\delta \mathscr{L}^{(d)}(x), \delta \Phi^{(d)}(x)$ determined.

We discuss these items in turn.
1. The correction terms $\delta \mathscr{L}^{(d)}(x)$ etc. have canonical mass dimension $4+d$. A path integral with weight $\mathrm{e}^{-\int \mathrm{d}^{4} x \mathscr{L}_{\text {eff }}(x)}$ is thus not renormalizable. Path integral expectation values are defined by expanding in the parameter $a$ before integrating over the fields. For our example, Eq. (2.5), we then have as a definition of $\delta C(x)$

$$
\begin{aligned}
\delta C(x) & =\delta C^{\mathscr{L}}(x)+\delta C^{\Phi}(x), \\
\delta C^{\mathscr{L}}(x-y) & =-\int \mathrm{d}^{4} z\left\langle\Phi(x) \Phi(y) \delta \mathscr{L}^{(2)}(z)\right\rangle_{\text {cont }}^{\text {con }}, \\
\delta C^{\Phi}(x-y) & =\langle\delta \Phi(x) \Phi(y)\rangle_{\text {cont }}^{\text {con }}+\langle\Phi(x) \delta \Phi(y)\rangle_{\text {cont }}^{\text {con }}
\end{aligned}
$$

where $\langle X\rangle_{\text {cont }}^{\text {con }}$ is given by the standard continuum connected correlation function with continuum Lagrangian

$$
\begin{aligned}
\mathscr{L}_{\text {cont }}(A) & =-\frac{1}{2 g_{0}^{2}} \sum_{\mu, \nu} \operatorname{tr}\left(F_{\mu \nu}(A) F_{\mu \nu}(A)\right), \\
F_{\mu \nu}(A) & =\left[D_{\mu}(A), D_{\nu}(A)\right],
\end{aligned}
$$

written in terms of the covariant derivative

$D_{\mu}(A)=\partial_{\mu}+A_{\mu}$.

We have already anticipated that 2 . leads to the vanishing of $\delta \mathscr{L}^{(1)}, \delta \Phi^{(1)}$ and used a shorthand $\delta \Phi=\delta \Phi^{(2)}$.

2 . The correction Lagrangians $\delta \mathscr{L}^{(d)}$ are linear combinations

$\delta \mathscr{L}^{(d)}(x)=\sum_{i} \omega_{i}\left(g_{0}^{2}\right) \mathcal{O}_{i}(x)$

of local operators $\mathcal{O}_{i}(x)$ which comply with the symmetries of the underlying lattice theory and have a mass dimension $4+d$. Gauge invariance is one of the symmetries (gauge fixing is needed only in Sect. 3 where we report on the perturbative computation). One may further drop all combinations of fields which vanish by the continuum equation of motion, $\left[D_{\mu}, F_{\mu \nu}(x)\right]=0,\left(\right.$ such as $\left.\mathcal{O}=\operatorname{tr}\left(\left[D_{\mu}, F_{\mu \nu}\right]\left[D_{\rho} F_{\rho \nu}\right]\right)\right)$ [12] as well as all operators which can be written as total derivatives of the form $\phi=\partial_{\mu} K_{\mu}$. After doing that, we have a so called "on-shell" basis. For YM it consists of two operators, which we may choose as

$\mathcal{O}_{1}=\frac{1}{g_{0}^{2}} \sum_{\mu, \nu, \rho} \operatorname{tr}\left(\left[D_{\mu}, F_{\nu \rho}\right]\left[D_{\mu}, F_{v \rho}\right]\right)$,

$\mathcal{O}_{2}=\frac{1}{g_{0}^{2}} \sum_{\mu, \nu} \operatorname{tr}\left(\left[D_{\mu}, F_{\mu \nu}\right]\left[D_{\mu}, F_{\mu \nu}\right]\right)$,

already known from Refs. $[15,16] .{ }^{4}$ Note that $\mathcal{O}_{2}$ breaks the $\mathrm{O}(4)$ rotational invariance of the continuum Lagrangian Eq. (2.10) down to $90^{\circ}$ rotations around the lattice axes.

\footnotetext{
4 That reference discusses the construction of a lattice improved action such that the $a^{2}$ terms in the SymEFT are absent. The basis of operators is the same.
} 
Dropping it, one has the general effective Lagrangian of a low energy theory with just gauge fields and $\mathrm{O}(4)$ invariance. This is a (tiny) sector of the Lagrangian considered for beyond the standard model phenomenology in Ref. [17]. The operator, $\frac{1}{g_{0}^{3}} \operatorname{tr}\left(F_{\mu \nu} F_{\nu \rho} F_{\rho \mu}\right)$, considered there is seen to be on-shell equivalent to

$$
\begin{aligned}
\mathcal{O}_{1}= & \frac{2}{g_{0}^{2}} \sum_{\mu, \nu, \rho}\left(\operatorname{tr}\left(\left[D_{\mu}, F_{\mu \nu}\right]\left[D_{\rho}, F_{\rho \nu}\right]\right)-\operatorname{tr}\left(F_{\mu \nu} F_{\nu \rho} F_{\rho \mu}\right)\right) \\
& +(\text { total divergences })
\end{aligned}
$$

using integration by parts and the Bianchi identity. Gauge invariant dimension five operators do not exist and thus YM theory has $n_{\min }=2$. The corrections to the continuum fields $\Phi_{i}$ will not be needed.

Now we consider the $a$ expansion of our observable,

$\mathcal{P}=\mathcal{P}^{\text {cont }}+a^{2}\left[\delta \mathcal{P}^{\mathscr{L}}+\delta \mathcal{P}^{\Phi}\right]+\mathrm{O}\left(a^{3}\right)$.

Inserting the spectral representations into the ratios $C_{i}^{\mathscr{L}} / C_{i}^{\text {cont }}$ which appear as one expands the r.h.s. of Eq. (2.2) in $a$, one sees $^{5}$

$\delta \mathcal{P}^{\mathscr{L}}=-\frac{1}{2}\left[\left\langle i\left|\delta \mathscr{L}^{(2)}(0)\right| i\right\rangle-\left\langle j\left|\delta \mathscr{L}^{(2)}(0)\right| j\right\rangle\right], \delta \mathcal{P}^{\Phi}=0$.

The states $|i\rangle,|j\rangle$ with $\langle i \mid i\rangle=\langle j \mid j\rangle=2 L^{3}$ are the ground states of the Hamiltonian of the finite volume theory with spatial volume $L^{3}$ in the zero momentum sector of the Hilbert space with the quantum numbers of $\Phi_{i}, \Phi_{j}$. The vanishing of $\delta \mathcal{P}^{\Phi}$ was to be expected as the energy of a physical state should not depend on the interpolating field used to create it, including its renormalization. Since physical quantities which do depend on $\delta \Phi$ have so far not been in the focus of lattice computations, and also because each field appearing in the correlation functions has to be considered separately, we will ignore the contribution of $\delta \Phi$ from now on. We concentrate on spectral quantities.

3 . The coefficients $\omega_{i}$ are needed, in particular their dependence on the parameters of the theory. Eq. (2.16) makes it clear that actually we first have to renormalize the operators $\mathcal{O}_{i}$ and then determine their coefficients by matching, which will be discussed in Sect. 4. Renormalization introduces a dependence on the renormalization scale $\mu$ (and scheme). By renormalization group improvement we turn it into a dependence on the lattice spacing, which we are seeking. In the 2-d $\mathrm{O}(\mathrm{N})$ sigma model, all this has been done to next-toleading order in the coupling [7]. Here we are content with the leading order since it predicts the asymptotic behavior of $\Delta \mathcal{P}$.

\footnotetext{
$\overline{5}$ For intermediate steps in the derivation, see [18], sect. 9.4.1. In quantum mechanics the relation given is the Feynman-Hellmann theorem.
}

Before proceeding it is convenient to switch to a basis of operators, with elements $\mathcal{B}_{i}=\sum_{j} v_{i j} \mathcal{O}_{j}$ which do not mix at one-loop order, i.e.

$\mathcal{B}_{i}^{\mathrm{R}}(\mu)=\left[1+g^{2} Z_{i}^{(1)}+\mathrm{O}\left(g^{4}\right)\right] \mathcal{B}_{i}$,

where $\mathcal{B}_{i}^{\mathrm{R}}(\mu)$ denote the renormalized operators in some scheme at renormalization scale $\mu$. One may think of the $\overline{\mathrm{MS}}$ scheme.

In general, we then have $\Delta_{\mathcal{P}}=\sum_{i} \bar{c}_{i} \mathcal{M}_{\mathcal{P}, i}^{\mathrm{R}}$, where at leading order in the coupling $\omega_{j}=\omega_{j}^{(n)} g_{0}^{2 n}+\mathrm{O}\left(g_{0}^{2 n+2}\right), \omega_{j}^{(n)}$ $=\sum_{i} \bar{c}_{i}^{(n)} v_{i j}$ and $\mathcal{M}_{\mathcal{P}, i}^{\mathrm{R}}$ are matrix elements of the operators $\mathcal{B}_{i}$ in the continuum field theory. The renormalized matrix elements are denoted

$\mathcal{M}_{\mathcal{P}, i}^{\mathrm{R}}(\mu)=\left\langle\psi_{\mathcal{P}}\left|\mathcal{B}_{i}^{\mathrm{R}}(\mu)\right| \psi_{\mathcal{P}}\right\rangle$,

with some physical state $\left|\psi_{\mathcal{P}}\right\rangle$, analogous to $|i\rangle,|j\rangle$, see Eq. (2.16). We have suppressed the spacetime argument of $\mathcal{B}_{i}$.

The coefficients $\bar{c}_{i}$ depend on the renormalization scheme adopted for $\mathcal{B}_{i}^{\mathrm{R}}$ as well as on $\mu$ and $a$. We may thus write (dropping higher powers of $a$ without notice)

$\Delta_{\mathcal{P}}(a)=-a^{2} \sum_{i} \bar{c}_{i}(\bar{g}(\mu), a \mu) \mathcal{M}_{\mathcal{P}, i}^{\mathrm{R}}(\mu)$,

where the dependence of $\bar{c}_{i}$ on $\mu$ cancels the one of $\mathcal{M}_{\mathcal{P}, i}^{\mathrm{R}}(\mu)$.

In order to systematically learn about the behavior for small $a$ we use renormalization group improvement, namely we set $\mu=1 / a$, and introduce the renormalization group invariant matrix elements

$\mathcal{M}_{\mathcal{P}, i}^{\mathrm{RGI}}=\sum_{j} \varphi_{i j}(\bar{g}(\mu)) \mathcal{M}_{\mathcal{P}, j}^{\mathrm{R}}(\mu)=\left\langle\psi_{\mathcal{P}}\left|\mathcal{B}_{i}^{\mathrm{RGI}}\right| \psi_{\mathcal{P}}\right\rangle$.

The matrix valued function (Pexp denotes path ordering: terms with smallest $x$ appear to the left)

$$
\begin{aligned}
\varphi(\bar{g}) & =\left[2 b_{0} \bar{g}^{2}\right]^{-\gamma^{(0)} / 2 b_{0}} \operatorname{Pexp}\left\{-\int_{0}^{\bar{g}} \mathrm{~d} x\left[\frac{\gamma(x)}{\beta(x)}-\frac{\gamma^{(0)}}{b_{0} x}\right]\right\} \\
& =\left[2 b_{0} \bar{g}^{2}\right]^{-\gamma^{(0)} / 2 b_{0}} \times\left[1+\mathrm{O}\left(\bar{g}^{2}\right)\right]
\end{aligned}
$$

involves the anomalous dimension matrix $\gamma$ defined by

$\mu \frac{\mathrm{d}}{\mathrm{d} \mu} \mathcal{B}_{i}^{\mathrm{R}}(\mu)=\sum_{j} \gamma_{i j}(\bar{g}(\mu)) \mathcal{B}_{j}^{\mathrm{R}}(\mu)$.

It has the expansion

$\gamma(\bar{g})=-\bar{g}^{2}\left[\gamma^{(0)}+\gamma^{(1)} \bar{g}^{2}+\cdots\right]$, 
where by our choice of basis $\gamma^{(0)}$ is diagonal,

$\frac{1}{2 b_{0}} \gamma^{(0)}=\operatorname{diag}\left(\hat{\gamma}_{1}, \hat{\gamma}_{2}\right)$.

Our convention for the $\beta$-function is $\beta(\bar{g}(\mu))=\mu \frac{\mathrm{d}}{\mathrm{d} \mu} \bar{g}(\mu)$ with expansion $\beta(\bar{g})=-\bar{g}^{3}\left(b_{0}+b_{1} \bar{g}^{2}+\cdots\right)$.

Asymptotic freedom means that perturbation theory is applicable at small $a$. The asymptotic behavior of Eq. (2.19) can thus be inferred from (renormalized) perturbation theory. The $\mathrm{O}\left(g^{2}\right)$ term in Eq. (2.22) is then subdominant and further we may expand

$\bar{c}_{i}\left(\bar{g}\left(a^{-1}\right), 1\right)=\bar{c}_{i}^{(0)}+\bar{c}_{i}^{(1)} \bar{g}^{2}\left(a^{-1}\right)+\cdots$.

Putting everything together and concentrating on the leading term we arrive at

$$
\begin{aligned}
\Delta_{\mathcal{P}}(a)= & -a^{2} \sum_{i} \bar{c}_{i}^{(0)}\left[2 b_{0} \bar{g}^{2}\left(a^{-1}\right)\right]^{\hat{\gamma}_{i}} \\
& \times \mathcal{M}_{\mathcal{P}, i}^{\mathrm{RGI}}\left[1+\mathrm{O}\left(\bar{g}^{2}\left(a^{-1}\right)\right]+\mathrm{O}\left(a^{4}\right) .\right.
\end{aligned}
$$

Ordering $\hat{\gamma}_{1}<\hat{\gamma}_{2}$, the leading asymptotics is

$\Delta_{\mathcal{P}}(a) \sim a^{2}\left[2 b_{0} \bar{g}^{2}\left(a^{-1}\right)\right]^{\hat{\gamma}_{1}} \sim a^{2}\left[\frac{1}{-\log (a \Lambda)}\right]^{\hat{\gamma}_{1}}$,

unless $\bar{c}_{1}^{(0)}$ or the matrix element $\mathcal{M}_{\mathcal{P}, 1}^{\mathrm{RGI}}$ vanish. Generically, there is no reason for the latter to do so. A positive/negative $\hat{\gamma}_{1}$ leads to an accelerated/decelerated asymptotic convergence as compared to naive $a^{2}$ behavior.

\section{One-loop computation of the anomalous dimension matrix}

We now turn to the anomalous dimension matrix $\gamma^{(0)}$. Although the renormalization of composite pure gauge theory operators has been discussed extensively $[17,19]$, a new computation is necessary because of the rotation symmetry violating operator $\mathrm{O}_{2}$, Eq. (2.13), which is not found in the literature. We thus employed dimensional regularization and computed the renormalization matrix,

$$
\left(\begin{array}{l}
\mathcal{O}_{1} \\
\mathcal{O}_{2}
\end{array}\right)_{\mathrm{R}}=\left(\begin{array}{cc}
Z_{11} & 0 \\
Z_{21} & Z_{22}
\end{array}\right)\left(\begin{array}{l}
\mathcal{O}_{1} \\
\mathcal{O}_{2}
\end{array}\right)
$$

to one-loop order. Here $Z_{12}$ vanishes because dimensional regularization preserves rotational symmetry and thus $\left(\mathcal{O}_{1}\right)_{\mathrm{R}}$ can not have a rotational non-invariant piece $\mathrm{Z}_{12} \mathrm{O}_{2}$.

The $Z$-matrix is obtained from a perturbative computation of a sufficient number of expectation values

$C_{i k}^{\mathcal{O}}=\left\langle\mathcal{O}_{i} \mathcal{O}_{k}^{\text {probe }}\right\rangle$ of the operators $\mathcal{O}_{i}$ together with suitable multi-local, renormalized, operators $\mathcal{O}_{k}^{\text {probe }}$. We may choose $\mathcal{O}_{k}^{\text {probe }}$ including their kinematics to simplify the computation. Unfortunately, just choosing them to be composed of local gauge invariant operators, e.g. $\operatorname{tr} F_{\mu \nu} F_{\mu \nu}$, one quickly discovers that one-loop computations are insufficient, since the tree-level correlation functions vanish.

As one option, we thus relaxed on manifest gauge invariance of $C_{i k}$ and consider gauge dependent Green's functions with

$$
\begin{aligned}
& \mathcal{O}_{1}^{\text {probe }}=\tilde{A}^{a}\left(p_{1}\right) \cdot \eta_{1} \tilde{A}^{b}\left(p_{2}\right) \cdot \eta_{2}, \\
& \mathcal{O}_{2}^{\text {probe }}=\tilde{A}^{a}\left(p_{1}\right) \cdot \eta_{1} \tilde{A}^{b}\left(p_{2}\right) \cdot \eta_{2} \tilde{A}^{c}\left(p_{3}\right) \cdot \eta_{3},
\end{aligned}
$$

in terms of the momentum space fields $\tilde{A}_{\mu}(p)$ $=\int \mathrm{d}^{4} x \mathrm{e}^{-i p x} A_{\mu}(x)$. We have $\sum_{i} p_{i}=-q$ as indicated in Fig. 1 and choose $\left[\left(p_{i}\right)_{0}\right]^{2}=-\left(\mathbf{p}_{i}\right)^{2}, p_{i} \cdot \eta_{i}=0$ for all $i$ with the Euclidean scalar product $p \cdot \eta=\sum_{\mu} p_{\mu} \eta_{\mu}$. In principle mixing of $\mathcal{O}_{i}$ with gauge-non-invariant operators then has to be taken into account [20,21]. However, those do not contribute to the on-shell Green's functions selected by our choice of kinematics. Since we want to restrict ourselves to the two and three gluon $\mathcal{O}^{\text {probe }}$ from above, we need to have a non-zero momentum $q$ of the operators $\mathcal{O}_{i}$. Otherwise the Green's functions vanish by kinematics. The price to pay is that $\mathcal{O}_{i}$ mix with the "total divergence operators",

$$
\begin{aligned}
& \phi_{1}=\frac{1}{g_{0}^{2}} \sum_{\mu, \nu, \rho} \partial_{\mu} \operatorname{tr}\left(F_{\rho \nu}\left[D_{\mu}, F_{\rho \nu}\right]\right), \\
& \phi_{2}=\frac{1}{g_{0}^{2}} \sum_{\mu, \nu} \partial_{\mu} \operatorname{tr}\left(F_{\mu \nu}\left[D_{\mu}, F_{\mu \nu}\right]\right),
\end{aligned}
$$

as

$$
\left(\begin{array}{l}
\mathcal{O} \\
\phi
\end{array}\right)_{\mathrm{R}}=\left(\begin{array}{cc}
Z & A^{\mathcal{O}} \boldsymbol{\phi} \\
0 & Z^{\mathscr{\Phi}}
\end{array}\right)\left(\begin{array}{l}
\mathcal{O} \\
\phi
\end{array}\right),
$$

with a block-triangular structure.

As a second option, we considered the background field method [22-25]. It consists of introducing a smooth classical background field, $B_{\mu}(x)$. The gauge field,

$A_{\mu}=B_{\mu}+g_{0} Q_{\mu}$,

is split into the background field and the quantum fluctuations $Q_{\mu}$. Note that the background field is not required to satisfy the equation of motion. In addition to the Lagrangian

$\mathscr{L}_{\mathrm{bf}}(B, q)=\mathscr{L}_{\text {cont }}\left(B+g_{0} Q\right)$,

one chooses the background field gauge with gauge-fixing term

$\mathscr{L}_{\mathrm{gf}}(B, Q)=-\lambda_{0} \sum_{\mu, \nu} \operatorname{tr}\left(\left[D_{\mu}(B), Q_{\mu}\right]\left[D_{\nu}(B), Q_{\nu}\right]\right.$ 


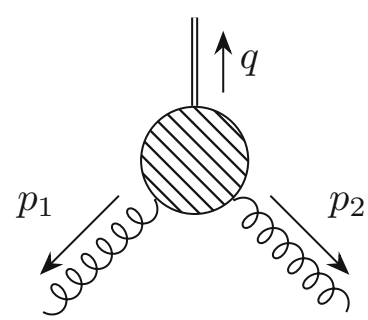

(a) Two-point function.

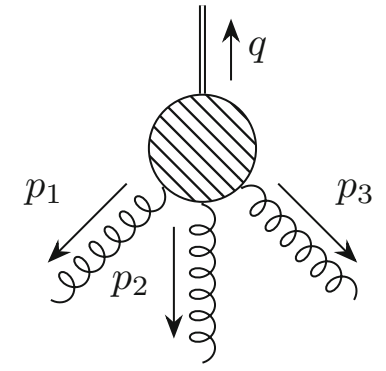

(b) Three-point function.
Fig. 1 Schematic representation of the needed two-point and threepoint functions with insertion of an operator $\mathcal{O}_{i}$. The "blob" represents all possible connected tree-level and one-loop graphs with given number of external legs

instead of the standard $-\lambda_{0} \operatorname{tr}\left(\left(\partial_{\mu} A_{\mu}\right)\left(\partial_{\nu} A_{\nu}\right)\right)$ and adds a Faddeev Popov term [26].

In this case, we can form

$$
\begin{aligned}
& \mathcal{O}_{1}^{\text {probe }}=\tilde{B}_{\mu}^{a}\left(p_{1}\right) \tilde{B}_{v}^{b}\left(p_{2}\right), \\
& \mathcal{O}_{2}^{\text {probe }}=\tilde{B}_{\mu}^{a}\left(p_{1}\right) \tilde{B}_{v}^{b}\left(p_{2}\right) \tilde{B}_{\rho}^{c}\left(p_{3}\right),
\end{aligned}
$$

just in terms of the background field, and obtain gauge invariant $C_{i k}$ by construction. We can remain with Euclidean momenta and do not need a nonzero momentum to flow into the operator $\mathcal{O}_{i}$. Thus the mixing with total divergence operators does not contribute any more. The downside is that here the equations of motion do not hold. Therefore, we have to consider the mixing structure

$$
\left(\begin{array}{l}
\mathcal{O} \\
\mathcal{E}
\end{array}\right)_{\mathrm{R}}=\left(\begin{array}{cc}
Z & A^{\mathcal{O} \mathcal{E}} \\
0 & Z^{\mathcal{E}}
\end{array}\right)\left(\begin{array}{l}
\mathcal{O} \\
\mathcal{E}
\end{array}\right)
$$

with the extra operator

$$
\mathcal{E}=\frac{1}{g_{0}^{2}} \sum_{\mu, \nu, \rho} \operatorname{tr}\left(\left[D_{\mu}, F_{\mu \nu}\right]\left[D_{\rho}, F_{\rho \nu}\right]\right) .
$$

Since we are just interested in the renormalization matrix $Z$, it suffices to consider only $\mathcal{O}_{\mathrm{R}}$, the first block row of the above equations. Those define the renormalized $\left(C_{i k}^{\mathcal{O}}\right)_{\mathrm{R}}$, replacing $\mathcal{O}$ with $\mathcal{O}_{\mathrm{R}}$. We write the resulting equations as

$\left(C_{i k}^{\mathcal{O}}\right)_{\mathrm{R}}=\sum_{j=1}^{2} Z_{i j} C_{j k}^{\mathcal{O}}+\sum_{l} A_{i l} C_{l k}^{\mathrm{red}}$,

where $C_{l k}^{\text {red }}$ is formed of the needed redundant operators which mix into $\mathcal{O}$. Without background field the redundant operators are $\boldsymbol{D}$. With background field there is just the operator $\mathcal{E}$. Expanding

$$
\begin{aligned}
Z_{i j} & =\delta_{i j}+\bar{Z}_{i j} \frac{g_{\mathrm{R}}^{2}}{\epsilon}+\mathrm{O}\left(\epsilon^{0}, g_{\mathrm{R}}^{4}\right), \\
A & =\bar{A} \frac{g_{\mathrm{R}}^{2}}{\epsilon}+\mathrm{O}\left(\epsilon^{0}, g_{\mathrm{R}}^{4}\right),
\end{aligned}
$$

$$
C_{i k}^{\mathcal{O}}=\left(C_{i k}^{\mathcal{O}}\right)^{(0)}+\overline{C_{i k}^{\mathcal{O}}} \frac{g_{\mathrm{R}}^{2}}{\epsilon}+\mathrm{O}\left(\epsilon^{0}, g_{\mathrm{R}}^{4}\right),
$$$$
C^{\mathrm{red}}=\left(C^{\mathrm{red}}\right)^{(0)}+\mathrm{O}\left(g_{\mathrm{R}}^{2}\right) \text {, }
$$

and requiring the finiteness of $\left(C_{i k}^{\mathcal{O}}\right)_{\mathrm{R}}$, the desired $\bar{Z}_{i j}$ (as well as $\bar{A}$ ) are obtained as the solution of the linear system of equations (each $i=1,2$ and all $k$ yield an equation),

$$
\sum_{j=1}^{2} \bar{Z}_{i j}\left(C_{j k}^{\mathcal{O}}\right)^{(0)}+\sum_{l} \bar{A}_{i l}\left(C_{l k}^{\mathrm{red}}\right)^{(0)}=-\overline{C_{i k}^{\mathcal{O}}} .
$$

There is one subtlety in applying the above. The equations assume that the observables $C_{j k}^{\mathcal{O}}$ are infrared finite. With the chosen on-shell kinematics in the first case, this is, however, not true and the $1 / \epsilon$ terms contain in principle a mix of ultraviolet and infrared divergences. Therefore we use the by now common following trick, called infrared rearrangement [2729]. For each loop integral, we rewrite the denominators in the form

$\frac{1}{(k+p)^{2}}=\frac{1}{k^{2}+\Omega}-\frac{2 k p+p^{2}-\Omega}{\left(k^{2}+\Omega\right)(k+p)^{2}}$,

where $k$ is the loop momentum and $\Omega$ is an arbitrary positive constant. The second term on the r.h.s. is one power less ultraviolet divergent and the first one has no source of infrared divergence. We can usually restrict ourselves to the first one since we are just interested in the ultraviolet divergences which determine the renormalization. If necessary, one can apply the transformation repeatedly. While for many integrals this trick is not necessary, we carry it out in all cases, since all integrals are then brought to the standard form $\int \mathrm{d}^{D} k\left[k^{2}+\Omega\right]^{-n} k_{\mu_{1}} \ldots k_{\mu_{l}}$ up to the finite and infrared divergent parts which we just drop. Note that the $Z$-factors are independent of $\Omega$. We have used this throughout as a check on our results.

The computation was carried out with the help of computer algebra packages. Feynman graphs were generated by QGRAF [30,31], formally treating the operator insertions with the help of additional non-propagating scalar fields, $\varphi_{i}(x)$, called "anchor", through additional terms $\sum_{i} \varphi_{i}(x) \mathcal{O}_{i}(x)$ in the Lagrangian. The Feynman rules were generated using FORM [32], which we also used for tricks such as Eq. (3.16), to reduce the Feynman graphs to standard one-loop integrals, and to isolate the $1 / \epsilon$ poles.

The computed two-point and three-point functions with operator insertions are shown schematically in Fig. 1. We checked explicitly that the results for both cases, non-zero $q$ vs. background field, agree. They read

$\bar{Z}=\frac{C_{\mathrm{A}}}{(4 \pi)^{2}}\left(\begin{array}{cc}7 / 3 & 0 \\ -7 / 15 & 21 / 5\end{array}\right)$.

The element $\bar{Z}_{11}$ agrees with the value found in the literature [33]. For completeness we also report the mixing 


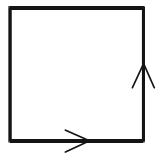

(0)

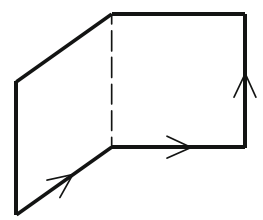

(2)

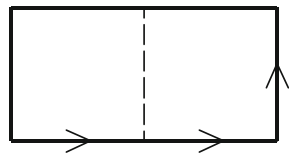

(1)

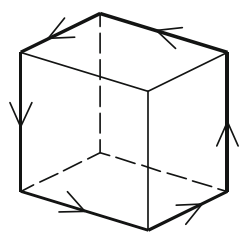

(3)
Fig. 2 Graphical representations [5] of the loop geometries contributing to commonly used lattice gauge actions

terms $\left(C_{\mathrm{A}}=\mathrm{N}\right.$ for gauge group $\left.\mathrm{SU}(\mathrm{N})\right)$

$$
\begin{aligned}
\bar{A}^{\mathcal{O}} & =\frac{C_{\mathrm{A}}}{(4 \pi)^{2}}\left(\begin{array}{cc}
-6 & 0 \\
-21 / 20 & -9 / 5
\end{array}\right), \\
\bar{A}^{\mathcal{O E}} & =\frac{C_{\mathrm{A}}}{(4 \pi)^{2}}\left(\begin{array}{c}
\frac{23}{6}-\frac{3}{2 \lambda_{\mathrm{R}}} \\
\frac{7}{15}-\frac{1}{2 \lambda_{\mathrm{R}}}
\end{array}\right), \\
Z^{\mathcal{E}} & =1+\frac{C_{\mathrm{A}}}{(4 \pi)^{2}}\left(\frac{5}{4}-\frac{3}{4 \lambda_{\mathrm{R}}}\right) \frac{g_{\mathrm{R}}^{2}}{\epsilon} .
\end{aligned}
$$

We read off that the choice of basis,

$\mathcal{B}_{1}=\mathcal{O}_{1}, \quad \mathcal{B}_{2}=-\frac{1}{4} \mathcal{O}_{1}+\mathcal{O}_{2}$,

renormalizes without mixing at one-loop order,

$$
\begin{aligned}
& \mathcal{B}_{i}^{\mathrm{R}}=\left[1+\bar{Z}_{i}^{\mathcal{B}} \frac{g_{\mathrm{R}}^{2}}{\epsilon}\right] \mathcal{B}_{i}+\mathrm{O}\left(g_{\mathrm{R}}^{4}\right), \\
& \bar{Z}_{1}^{\mathcal{B}}=\frac{7}{3} \frac{1}{(4 \pi)^{2}}, \quad \bar{Z}_{2}^{\mathcal{B}}=\frac{21}{5} \frac{1}{(4 \pi)^{2}} .
\end{aligned}
$$

The anomalous dimensions of Eq. (2.25) are ${ }^{6}$

$$
\hat{\gamma}_{1}=7 / 11 \approx 0.636, \quad \hat{\gamma}_{2}=63 / 55 \approx 1.145 \text {, }
$$

independent of the number of colors.

\section{Matching to lattice actions}

The final ingredient needed to predict the form of the cutoff effects are the coefficients of the higher dimensional operators in the effective Lagrangian, step "3." in Sect. 2. At leading order of perturbation theory considered here, we just need the lowest order coefficients $\bar{c}_{i}^{(0)}$ of the functions $\bar{c}_{i}$,

$\overline{6 \text { At one-loop }}$ order we have $\gamma_{i}=2 b_{0} \hat{\gamma}_{i}=\bar{Z}_{i}^{\mathcal{B}}$.
Table 1 Commonly used gauge actions and their coefficients of the operators $\mathcal{B}_{1}, \mathcal{B}_{2}$ in the SymEFT. The row "Symanzik improved" applies to all actions with leading order in $g_{0}^{2}$ coefficients as specified there

\begin{tabular}{llllll}
\hline Action & $e_{1}$ & $e_{2}$ & $e_{3}$ & $\bar{c}_{1}^{(0)}$ & $\bar{c}_{2}^{(0)}$ \\
\hline Wilson, Eq. (2.1) & 0 & 0 & 0 & $\frac{1}{48}$ & $\frac{1}{12}$ \\
Symanzik improved & $-\frac{1}{12}$ & 0 & 0 & 0 & 0 \\
Iwasaki [34] & -0.331 & 0 & 0 & -0.0619 & -0.2477 \\
DBW2 [35,36] & -1.4088 & 0 & 0 & -0.3314 & -1.3255 \\
\hline
\end{tabular}

Eq. (2.26). At tree-level, no divergences occur in the path integral. One may therefore perform a naive classical expansion of the lattice action in $a$, setting $U(x, \mu)=\mathrm{e}^{a A_{\mu}(x)}$ with a smooth continuum gauge field $A_{\mu}$. This expansion has been carried out by Lüscher and Weisz [16] for a set of gauge actions, in particular for those consisting of the lattice loops depicted in Fig. 2. For each of these loops one sums over all lattice points corresponding to the lower left corners in the graph and over all orientations on the lattice, e.g. for the plaquette term ( 0 ) one sums over $\mu>v$, for the rectangle (1) over $\mu \neq v$ etc. There are $6,12,16,48$ orientations for the loops (0), (1), (2), (3). Apart from the overall pre-factor $2 / g_{0}^{2}$, we denote their coefficients at $g_{0} \rightarrow 0$ as $e_{i}, i=0,1,2,3$ (in Ref. [16] they are denoted $c_{i}(0)$ ). With

$e_{0}+8 e_{1}+8 e_{2}+16 e_{3}=1$,

the leading term in the $a$-expansion,

$S_{\text {lat }}^{\text {class }}=\int \mathrm{d}^{4} x\left\{\mathscr{L}_{\text {cont }}(x)+a^{2} \sum_{i=1}^{2} \omega_{i} \mathcal{O}_{i}(x)+\cdots\right\}$,

has the conventional normalization. The ellipses summarize terms that vanish upon the use of the equation of motion and higher orders in $a$. From table 2 of [16] we find

$\bar{c}_{1}^{(0)}=\omega_{1}^{(0)}+\frac{1}{4} \omega_{2}^{(0)}=\frac{1}{48}+\frac{1}{4} e_{1}+\frac{1}{3} e_{2}-\frac{1}{4} e_{3}$,

$\bar{c}_{2}^{(0)}=\omega_{2}^{(0)}=\frac{1}{12}+e_{1}-e_{3}$.

The standard Wilson plaquette action, Eq. (2.1), has $e_{0}=$ $1, e_{1}=e_{2}=e_{3}=0$ and both $\mathcal{B}_{1}$ and $\mathcal{B}_{2}$ contribute to the order $a^{2}$. Symanzik improved actions have $\bar{c}_{i}^{(0)}=0$ by design. Other actions such as the Iwasaki action and the "DBW2" action lead to quite large coefficients. We show a summary in Table 1. All considered lattice actions just have the plaquette and the rectangle terms. This turns out to lead to vanishing coefficients $e_{2}, e_{3}$ and in the classical $a^{2}$ expansion only $\mathcal{O}_{1}$ contributes in the $\mathcal{O}_{i}$ basis [16]. As discussed before we have to go to the basis $\mathcal{B}_{i}$ with diagonal renormalization at one-loop. The relevant coefficients for the asymptotics are then related by $\bar{c}_{2}^{(0)}=4 \bar{c}_{1}^{(0)}$. 


\section{Examples for the asymptotic behavior}

For convenience we combine here the main results of the previous two sections and discuss some interesting sample applications.

\subsection{Generic form for spectral quantities}

The cases considered in Table 1 are probably the most relevant for the Yang-Mills theory. Since they all satisfy $\bar{c}_{2}^{(0)}=4 \bar{c}_{1}^{(0)}$, we have the form

$$
\begin{aligned}
\Delta_{\mathcal{P}}(a) \\
=-a^{2} \bar{c}_{1}^{(0)}\left\{\left[2 b_{0} \bar{g}^{2}\left(a^{-1}\right)\right]^{\hat{\gamma}_{1}} \mathcal{M}_{\mathcal{P}, 1}^{\mathrm{RGI}}+4\left[2 b_{0} \bar{g}^{2}\left(a^{-1}\right)\right]^{\hat{\gamma}_{2}} \mathcal{M}_{\mathcal{P}, 2}^{\mathrm{RGI}}\right\} \\
\quad \times\left[1+\mathrm{O}\left(\bar{g}^{2}\left(a^{-1}\right)\right]\right. \text { for Wilson, Iwasaki, DBW2 actions. }
\end{aligned}
$$

The entire computed leading behavior only depends on the coefficient $\bar{c}_{1}^{(0)}$. While we cannot predict the relative contribution of the two powers $\hat{\gamma}_{1}, \hat{\gamma}_{2}$ because they depend on the non-perturbative matrix elements $\mathcal{M}^{\mathrm{RGI}}$, their mixture is the same for any of the three different actions. The only action dependence is in the coefficient of the rectangle term (geometry (1) of Fig. 2) and thus the leading cutoff effects have a relative size

Wilson : Iwasaki : DBW2 $\approx 1:(-3):(-16)$.

For a Symanzik improved action, the property $\bar{c}_{2}^{(0)}=$ $\bar{c}_{1}^{(0)}=0$ and additionally for a one-loop improved action $\bar{c}_{2}^{(1)}=\bar{c}_{1}^{(1)}=0$ means

$$
\begin{aligned}
\Delta_{\mathcal{P}}(a)= & -a^{2} \sum_{i} \bar{c}_{i}^{\left(n_{\mathrm{I}}\right)}\left[\bar{g}^{2}\left(a^{-1}\right)\right]^{n_{\mathrm{I}}}\left[2 b_{0} \bar{g}^{2}\left(a^{-1}\right)\right]^{\hat{\gamma}_{i}} \mathcal{M}_{\mathcal{P}, i}^{\mathrm{RGI}} \\
& \times\left[1+\mathrm{O}\left(\bar{g}^{2}\left(a^{-1}\right)\right]\right.
\end{aligned}
$$

where $n_{\mathrm{I}}=1$ for a tree-level improved action and $n_{\mathrm{I}}=2$ for a one-loop improved action and $n_{\mathrm{I}}=0$ without perturbative improvement. We illustrate the $a$ behavior in Fig. 3. One notices that over a typical range of $a$ from $a=0.1 \mathrm{fm}$ to $a=$ $0.04 \mathrm{fm}$, one has $20,40,60 \%$ (for $n_{\mathrm{I}}=0,1,2$, respectively) reductions of $\Delta_{\mathcal{P}}(a)$ as compared to the naive $a^{2}$ behavior.

We remind the reader, that gradient flow observables are excluded and that we have restricted ourselves to energy levels.

\subsection{Short distance observables}

Let us now consider the special case of a dimensionless short distance observable depending on a single physical length scale $r$. A simple example is $\mathcal{P}_{\mathrm{F}}=\frac{4 \pi}{C_{\mathrm{F}}} r^{2} F(r)$, with $F(r)$ the force between static quarks assumed here to be defined in terms of a discrete derivative of the potential which is correct up to order $a^{4}$ errors. $^{7}$ In particular, we are interested in the region of small $r$, which has two consequences. The ratio $a / r$ which determines the discretization errors is not as small as in the large distance region. The discussion of discretization errors is thus particularly important. Second, not only the continuum $\mathcal{P}(\Lambda r, 0)$ can be expanded in perturbation theory, but also the quantity at finite $a / r$ - both in lattice theory and in SymEFT. We want to summarize what one can learn from this.

The perturbative expansion in the lattice theory is expected to be of the form $[1,38]$

$$
\begin{aligned}
& \Delta_{\mathcal{P}}(\Lambda r, a / r) \\
& =\mathcal{P}(\Lambda r, a / r)-\mathcal{P}(\Lambda r, 0) \\
& =\mathcal{P}(\Lambda r, 0)\left[\delta_{0}(a / r)+\delta_{1}(a / r) \bar{g}_{\text {lat }}^{2}\left(r^{-1}\right)+\cdots\right] \\
& \quad \delta_{l}(a / r)=\frac{a^{2}}{r^{2}} \sum_{k=0}^{l} p_{l k} \log (a / r)^{k}+\mathrm{O}\left((a / r)^{4}\right) .
\end{aligned}
$$

On the other hand in SymEFT with renormalization group improvement, dropping the $\mathrm{O}\left(\bar{g}_{\text {lat }}^{2}\left(a^{-1}\right)\right)$ corrections, we have

$$
\begin{gathered}
\Delta_{\mathcal{P}}(\Lambda r, a / r)=-\frac{a^{2}}{r^{2}} \sum_{i} \bar{c}_{i}^{(0)}\left[2 b_{0} \bar{g}_{\text {lat }}^{2}\left(a^{-1}\right)\right]^{\hat{\gamma}_{i}}\left[r^{2} \mathcal{M}_{\mathcal{P}, i}^{\mathrm{RGI}}(r)\right] \\
=-\frac{a^{2}}{r^{2}} \mathcal{P}(\Lambda r, 0) \sum_{i} \bar{c}_{i}^{(0)}\left[\frac{\bar{g}_{\text {lat }}^{2}\left(a^{-1}\right)}{\bar{g}_{\text {lat }}^{2}\left(r^{-1}\right)}\right]^{\hat{\gamma}_{i}} K_{i}(r), \\
K_{i}(r)=\frac{r^{2} \mathcal{M}_{\mathcal{P}, i}^{\mathrm{R}}(r ; \mu)}{\mathcal{P}(\Lambda r, 0)}, \quad \mu=r^{-1},
\end{gathered}
$$

where the second argument $\mu$ in $\mathcal{M}^{\mathrm{R}}$ is the renormalization scale of the operator $\mathcal{B}_{i}^{\mathrm{R}}$.

For comparison to the fixed order perturbation theory form Eq. (5.4) we expand (remember $\left.\hat{\gamma}_{i}=\gamma_{i}^{(0)} /\left(2 b_{0}\right)\right)$

$$
\begin{gathered}
{\left[\frac{\bar{g}_{\text {lat }}^{2}\left(a^{-1}\right)}{\bar{g}_{\text {lat }}^{2}\left(r^{-1}\right)}\right]^{\hat{\gamma}_{i}}=1+\gamma_{i}^{(0)} \log (a / r) \bar{g}_{\text {lat }}^{2}\left(r^{-1}\right)+\mathrm{O}\left(\bar{g}_{\text {lat }}^{4}\right),} \\
K_{i}(r)=\left[K_{i}^{(0)}+K_{i}^{(1)} \bar{g}_{\text {lat }}^{2}\left(r^{-1}\right)+\mathrm{O}\left(\bar{g}_{\text {lat }}^{4}\right)\right]
\end{gathered}
$$

and find

$$
\begin{aligned}
& p_{00}=-\sum_{i} \bar{c}_{i}^{(0)} K_{i}^{(0)} \\
& p_{10}=-\sum_{i} \bar{c}_{i}^{(0)} K_{i}^{(1)}-\sum_{i} \bar{c}_{i}^{(1)} K_{i}^{(0)}
\end{aligned}
$$

\footnotetext{
7 Otherwise, if $\mathrm{O}\left(a^{2}\right)$ errors are associated with the definition of the lattice derivative, these can be taken into account explicitly.
} 

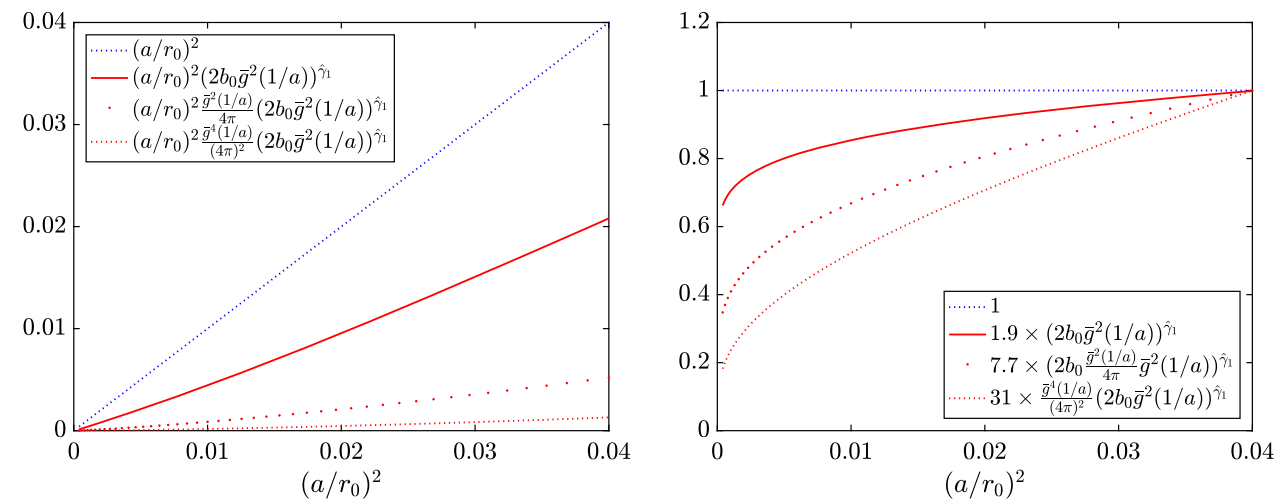

Fig. 3 Illustration of discretization errors, $\Delta_{\mathcal{P}}(a)$, Eq. (5.3) compared to naive $a^{2}$ behavior. We use $\alpha\left(5 / r_{0}\right)=\bar{g}^{2}\left(5 / r_{0}\right) / 4 \pi=0.25$, where $r_{0} \approx 0.5 \mathrm{fm} \mathrm{[37]} \mathrm{and} \mathrm{set} \mathrm{matrix} \mathrm{elements} \mathrm{to} \mathrm{one} \mathrm{in} \mathrm{units} \mathrm{of} r_{0}$ and set $c_{1}^{(i)}=1$. On the right, we drop the overall naive power of $a^{2} / r_{0}^{2}$ and normalize at $a / r_{0}=1 / 5$ such that the shape is clearly visible

$$
p_{11}=-\sum_{i} \bar{c}_{i}^{(0)} K_{i}^{(0)} \gamma_{i}^{(0)}
$$

This demonstrates the standard use of EFT in the perturbative domain. The EFT description and computation is more efficient since first of all it provides renormalization group improvement (1.h.s. of Eq. (5.8)) and second even the computation of coefficients $p_{l k}$ may be simplified. Apart from the one-loop matching coefficients of the action, $\bar{c}_{i}^{(1)}$, which can be computed by matching any convenient set of observables, only continuum perturbation theory quantities appear on the r.h.s. of Eqs. (5.10) and (5.11).

\section{Improved observables}

For short distance observables it is rather common to attempt a reduction of lattice spacing effects at the level of the expectation values rather than at the level of the action. For the static potential or $\mathcal{P}_{\text {F }}$, we refer the reader to [37,39]. Examples with higher orders in perturbation theory and with a combination of improvement of action and observable are found for example in [40-43].

To illustrate what is gained by considering SymEFT, it is sufficient to define a tree-level improved short distance observable,

$$
\begin{aligned}
\mathcal{P}^{\mathrm{impr}}(\Lambda r, a / r) & =\frac{\mathcal{P}(\Lambda r, a / r)}{1+\delta_{0}(a / r)} \\
& =\frac{\mathcal{P}(\Lambda r, a / r)}{1-\frac{a^{2}}{r^{2}} \sum_{i} \bar{c}_{i}^{(0)} K_{i}^{(0)}}+\mathrm{O}\left(a^{4} / r^{4}\right) .
\end{aligned}
$$

By construction, cutoff effects in fixed order perturbation theory are then suppressed by one power of $\bar{g}_{\text {lat }}^{2}$ (all orders in $a / r)$ and therefore also the coefficient $p_{00}$ of $a^{2} / r^{2}$ vanishes irrespective of the action. However, this neither means that the leading term $(i=1)$ in Eq. (5.6) vanishes nor that the sum of the two $\mathrm{O}\left(a^{2}\right)$ terms does. The sum of the two terms vanishes only for $a=r$, which is not at all where the $a^{2}$ expansion is applicable. In fact, inserting the denominator in Eqs. (5.13) into (5.6) one obtains

$$
\begin{aligned}
& \Delta_{\mathcal{P} \text { impr }}(\Lambda r, a / r) \\
& =-\frac{a^{2}}{r^{2}} \mathcal{P}(\Lambda r, 0) \sum_{i}\left\{\left[\frac{\bar{g}_{\text {lat }}^{2}\left(a^{-1}\right)}{\bar{g}_{\text {lat }}^{2}\left(r^{-1}\right)}\right]^{\hat{\gamma}_{i}}-1\right\} K_{i}^{(0)} \bar{c}_{i}^{(0)} .
\end{aligned}
$$

The effect of tree level improvement is the subtraction of the 1 in the curly bracket. For intermediate $a / r$, this will reduce the magnitude (and change the sign) of each term in the sum over $i$. However, asymptotically, for very small $a / r$, the tree level improvement leads to an increase of the $a^{2}$ effects. This behavior is tied to the sign of the $\hat{\gamma}_{i}$. For negative $\hat{\gamma}_{i}$, we would always have a reduction of the magnitude of the terms.

Usually the terms $K_{i}^{(0)} \bar{c}_{i}^{(0)}$ are known individually and one can divide out the complete leading order term,

$$
\mathcal{P}^{\mathrm{RG}-\mathrm{impr}}=\frac{\mathcal{P}}{1-\frac{a^{2}}{r^{2}} \sum_{i}\left[\frac{\bar{g}_{\text {lat }}^{2}\left(a^{-1}\right)}{\bar{g}_{\text {lat }}^{2}\left(r^{-1}\right)}\right]^{\hat{\gamma}_{i}} K_{i}^{(0)} \bar{c}_{i}^{(0)}},
$$

and have a renormalisation group and tree level improved observable. It then has leading corrections which are truly of order $\Delta_{\mathcal{P}} / \mathcal{P} \sim \frac{a^{2}}{r^{2}} \bar{g}_{\text {lat }}^{2}\left(r^{-1}\right)\left[\frac{\bar{g}_{\text {lat }}^{2}\left(a^{-1}\right)}{\bar{g}_{\text {lat }}^{2}\left(r^{-1}\right)}\right]^{\hat{\gamma}_{1}}$ as the name tree level improvement suggests.

We return to $\mathcal{P}_{\mathrm{F}}$. In this special case, the $\mathrm{O}(4)$ invariant operator $\mathcal{O}_{1}=\mathcal{B}_{1}$ does not contribute at tree level, $K_{1}^{(0)}=0$. Specializing to the Wilson plaquette action and the force along a lattice axes, we have $\bar{c}_{2}^{(0)}=1 / 12$ and $K_{2}^{(0)}=-9$. If one chooses a different direction on the lattice, e.g. a bodydiagonal, the matrix element $K_{2}^{(0)}$ is smaller, but the finite 


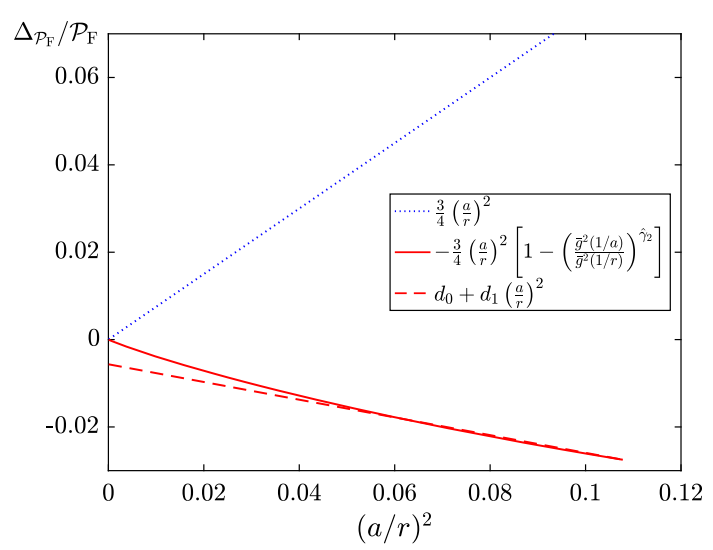

Fig. 4 Leading order discretization errors, $\Delta_{\mathcal{P}_{\mathrm{F}}}(a) / \mathcal{P}_{\mathrm{F}}$ of the static force, see the text. We use $\alpha(1 / r)=\bar{g}_{\text {lat }}^{2}(1 / r) /(4 \pi)=0.2$, and $K_{1}^{(0)}=0, K_{2}^{(0)} \bar{c}_{2}^{(0)}=-3 / 4$, corresponding to the Wilson plaquette action and the force along a lattice axes. The dotted line represents fixed order perturbation theory, the full line the remainder (on top of fixed order) predicted by SymEFT, and the dashed line shows a rough approximation, linear in $a^{2}$, to that latter

difference defining the force on the lattice has a larger discretization length. The various terms are illustrated in Fig. 4. The dotted line is the fixed order perturbation theory for $\Delta_{\mathcal{P}_{\mathrm{F}}} / \mathcal{P}_{\mathrm{F}}$ and the full curve the remainder, Eq. (5.14). The dashed line shows a rough linear approximation to the latter at large $a$. It extrapolates to a small value of $-0.6 \%$ at $a=0$. We may think of this as an example for the relative error one makes by approximating the cutoff effects of the tree-level improved observable linearly in $a^{2} .{ }^{8}$ Interpreting $\mathcal{P}_{\mathrm{F}}$ as a running coupling as explained for example in [44], this intercept represents a systematic (relative) uncertainty on the coupling. It translates into an about $1.5 \%$ error in the $\Lambda$-parameter of the theory, which is not entirely irrelevant given today's precision of results for it. Needless to say that the full logarithmic term Eq. (5.14) is better eliminated by use of Eq. (5.15).

\section{Schrödinger functional}

Short distance observables of particular interest can be defined in the Schrödinger functional [45]. Fixed order perturbation theory has been used extensively to study discretization errors in this environment. Here we consider their renormalization group improvement with the help of the SymEFT. We just consider the pure gauge theory and the Schrödinger functional with an abelian background field, where - as we will see - we do not have to deal with operator mixing.

\footnotetext{
${ }^{8}$ Usually the tree-level improved force is defined through an improved distance [37] $r_{\mathrm{I}}$. At the level of $a^{2}$ terms this is equivalent to Eq. (5.13).
}

In the lattice regularization, the Schrödinger functional can be defined by a path integral with action,

$$
\begin{aligned}
S_{\text {lattice }}^{\mathrm{SF}}= & \frac{2}{g_{0}^{2}} \sum_{0 \leq x_{0} \leq T-a} \sum_{\mathbf{x}} \sum_{\mu>v=0}^{3} p(x, \mu, v) \\
& +a\left(c_{\mathrm{t}}\left(g_{0}\right)-1\right) a^{3} \sum_{\mathbf{x}}\left[\mathcal{O}_{\mathrm{b}}^{1}(0, \mathbf{x})+\mathcal{O}_{\mathrm{b}}^{1}(T-a, \mathbf{x})\right],
\end{aligned}
$$

with

$$
\mathcal{O}_{\mathrm{b}}^{1}\left(x_{0}, \mathbf{x}\right)=\frac{2}{g_{0}^{2}} \frac{1}{a^{4}} \sum_{k=1}^{3} p(x, k, 0) \text {. }
$$

Space-time is a cylinder in the sense that we have periodic boundary conditions in space with period $L$ and Dirichlet boundary conditions on the time-slices $x_{0}=0$ and $x_{0}=T$, $\left.U(x, k)\right|_{x_{0}=0}=\mathrm{e}^{a C_{k}(L, \eta)},\left.\quad U(x, k)\right|_{x_{0}=T}=\mathrm{e}^{a C_{k}^{\prime}(L, \eta)}$.

For details we refer to [45], but we note that the dimensionless quantity $L C_{k}(L, \eta)$ is just a function of the dimensionless parameter $\eta$ (and a here irrelevant second parameter $v$ ) and that the field strength $F_{k l}$ vanishes at the two boundaries.

Under these conditions, which have been imposed for all numerical applications so far, the SymEFT for the YangMills Schrödinger functional is given by the formal continuum action

$$
\begin{aligned}
& S_{\mathrm{eff}}^{\mathrm{SF}}=\int \mathrm{d}^{3} \mathbf{x}\left\{\int_{0}^{T} \mathrm{~d} x_{0} \mathscr{L}_{\text {cont }}(x)+a \omega_{\mathrm{b}}\left[\mathcal{O}_{\mathrm{b}}(0, \mathbf{x})+\mathcal{O}_{\mathrm{b}}(T, \mathbf{x})\right]\right\} \\
& \quad+\mathrm{O}\left(a^{2}\right)
\end{aligned}
$$

with

$\mathcal{O}_{\mathrm{b}}(x)=-\frac{1}{g_{0}^{2}} \operatorname{tr}\left(F_{0 k}(x) F_{0 k}(x)\right)$.

The presence of the boundary terms in Eq. (6.4) is the reason for including the corresponding extra term proportional to $c_{\mathrm{t}}$ in the lattice formulation: the coefficients $c_{\mathrm{t}}^{(i)}$ in

$c_{\mathrm{t}}\left(g_{0}\right)=c_{\mathrm{t}}^{(0)}+c_{\mathrm{t}}^{(1)} g_{0}^{2}+\mathrm{O}\left(g_{0}^{4}\right)$,

can be chosen such that $\omega_{\mathrm{b}}$ vanishes and there are no linear terms in $a$ in the lattice Schrödinger functional at the corresponding order in perturbation theory [45].

A prominent observable in the Schrödinger functional is the running coupling

$\bar{g}^{-2}\left(L^{-1}\right)=\frac{1}{k}\left\langle S^{\prime}\right\rangle, \quad$ with $S^{\prime}=\left.\frac{\partial S}{\partial \eta}\right|_{\eta=0}$.

where $k=12 \pi$ imposes the standard normalization of the coupling, $\bar{g}^{2}=g_{0}^{2}+\mathrm{O}\left(g_{0}^{4}\right)+\mathrm{O}(a / L)$. We want to discuss 
the $a$-effects of $\bar{g}^{2}$ as an example. The definition of the $a$ effects requires to first renormalize. We here do this by lattice minimal subtraction,

$$
\begin{aligned}
& \bar{g}_{\text {lat }}^{2}(\mu)=Z_{\mathrm{g}}\left(\bar{g}_{\text {lat }}, a \mu\right) g_{0}^{2}, \\
& Z_{\mathrm{g}}\left(\bar{g}_{\text {lat }}, a \mu\right)=1-2 b_{0} \log (a \mu) \bar{g}_{\text {lat }}^{2}(\mu)+\mathrm{O}\left(g^{4}\right) .
\end{aligned}
$$

We can then define the function

$$
K\left(\bar{g}_{\text {lat }}^{2}\left(L^{-1}\right), a / L\right)=\bar{g}^{-2},
$$

which relates the renormalized couplings of the two schemes. It has a continuum limit and discretization errors

$$
\Delta K\left(\bar{g}_{\text {lat }}^{2}, a / L\right)=K\left(\bar{g}_{\text {lat }}^{2}, a / L\right)-K\left(\bar{g}_{\text {lat }}^{2}, 0\right) .
$$

They have the expansion

$$
\begin{aligned}
& \frac{\Delta K\left(\bar{g}_{\text {lat }}^{2}, a / L\right)}{K\left(\bar{g}_{\text {lat }}^{2}, 0\right)} \\
& =\frac{a}{L}\left[p_{00}+\left(p_{10}+p_{11} \log (a / L)\right) \bar{g}_{\text {lat }}^{2}\left(L^{-1}\right)+\mathrm{O}\left(g^{4}\right)\right] \\
& \quad+\mathrm{O}\left((a / L)^{2}\right),
\end{aligned}
$$

where analogously to before SymEFT predicts

$$
p_{11}=\gamma_{\mathrm{b}}^{(0)} p_{00} \text {. }
$$

An explicit one-loop computation [46] showed that

$p_{00}=0$ for $c_{\mathrm{t}}^{(0)}=1$,

$p_{10}=2 \times\left(c_{\mathrm{t}}^{(1)}+0.0890(2)\right)$ for $c_{\mathrm{t}}^{(0)}=1$.

Thus $c_{\mathrm{t}}^{(0)}=1, c_{\mathrm{t}}^{(1)}=-0.0890(2)$ leads to the absence of linear $a$-effects at one-loop. For this reason the perturbative computations have been carried out with $c_{\mathrm{t}}^{(0)}=1$ and from the published one-loop computation we do not have access to $\gamma_{\mathrm{b}}^{(0)}$. We note that general $c_{\mathrm{t}}^{(0)}$ was considered in [47] in the context of aspect ratios $T / L \neq 1$, but again $\gamma_{\mathrm{b}}^{(0)}$ can't be extracted because in the one-loop computation $c_{\mathrm{t}}^{(0)}$ was set such that $p_{00}=0$.

As was done in Sect. 3, the standard way to compute $\gamma_{\mathrm{b}}^{(0)}$ is to compute the one-loop renormalization of $\mathcal{O}_{\mathrm{b}}$. Here we extract it indirectly from the results of the two-loop computation of $[48,49]$. In contrast to Sect. 3 the computation thus relies entirely on the lattice regularization. Consider Eq. (6.1) with a lattice spacing $a \rightarrow a_{\mathrm{f}}$ and then replace

$c_{\mathrm{t}}\left(g_{0}\right)-1 \rightarrow \zeta$.

In this way $\zeta$ acts as a source for the lattice regularized operator $\mathcal{O}_{\mathrm{b}}$. The continuum function $K\left(\bar{g}_{\text {lat }}^{2}, 0\right)$ is given by

$$
K\left(\bar{g}_{\text {lat }}^{2}, 0\right)=\lim _{a_{\mathrm{f}} \rightarrow 0}\left[\left\langle S^{\prime}\right\rangle_{a_{\mathrm{f}}}\right]_{\zeta=0}
$$

and the first order correction in $a$ by

$$
\Delta K\left(\bar{g}_{\text {lat }}^{2}, a / L\right)=a \lim _{a_{\mathrm{f}} \rightarrow 0}\left[\frac{1}{a_{\mathrm{f}}} \frac{\partial}{\partial \zeta}\left\langle S^{\prime}\right\rangle_{a_{\mathrm{f}}}^{\mathrm{R}}\right]_{\zeta=0}+\mathrm{O}\left((a / L)^{2}\right) .
$$

The right hand side of Eq. (6.17) is the SymEFT prediction written as the continuum limit of the lattice regularized theory (with spacing $a_{\mathrm{f}}$ to distinguish it from $a$ ). Renormalization is indicated by the superscript R. In addition to Eq. (6.8) it affects the boundary operator $\mathcal{O}_{\mathrm{b}}$,

$$
\begin{aligned}
& \mathcal{O}_{\mathrm{b}}^{\text {lat }}=Z_{\mathrm{b}}\left(\bar{g}_{\text {lat }}, a_{\mathrm{f}} \mu\right) \mathcal{O}_{\mathrm{b}}, \\
& Z_{\mathrm{b}}\left(\bar{g}_{\text {lat }}, a_{\mathrm{f}} \mu\right)=1-\gamma_{\mathrm{b}}^{(0)} \log \left(a_{\mathrm{f}} \mu\right) \bar{g}_{\text {lat }}^{2}(\mu)+\cdots .
\end{aligned}
$$

We are now ready to extract $\gamma_{b}^{(0)}$ from the two-loop expansion,

$$
\begin{aligned}
\bar{g}^{-2} & =g_{0}^{-2}\left[1+k_{1} g_{0}^{2}+k_{2} g_{0}^{4}+\mathrm{O}\left(g_{0}^{6}\right)\right] \\
k_{1} & =-m_{1}^{\mathrm{a}}+c_{\mathrm{t}}^{(1)} \frac{2 a_{\mathrm{f}}}{L}, \\
k_{2} & =-m_{2}^{\mathrm{a}}-c_{\mathrm{t}}^{(1)} m_{2}^{\mathrm{b}}-\left(c_{\mathrm{t}}^{(1)}\right)^{2} m_{2}^{\mathrm{c}}-c_{\mathrm{t}}^{(2)} m_{2}^{\mathrm{d}},
\end{aligned}
$$

derived in $[48,49]$ for $c_{\mathrm{t}}^{(0)}=1$. We use the asymptotic expansion of the coefficients $m_{i}^{k}$ in powers of $\frac{a_{\mathrm{f}}}{L}$ and $\log \left(a_{\mathrm{f}} / L\right)$ given in Ref. $[48,49]$ and keep the notation of the coefficients of these references. But first we note that with $\left\langle S^{\prime}\right\rangle=k / \bar{g}^{2}$ we have

$$
\begin{aligned}
\frac{1}{a_{\mathrm{f}}} & {\left[\frac{\partial}{\partial \zeta}\left\langle S^{\prime}\right\rangle_{a_{\mathrm{f}}}\right]_{\zeta=0}^{\mathrm{R}} } \\
= & \frac{1}{a_{\mathrm{f}}} Z_{\mathrm{b}}\left(\bar{g}_{\text {lat }}, a_{\mathrm{f}} \mu\right)\left[\frac{\partial}{\partial \zeta}\left\langle S^{\prime}\right\rangle_{a_{\mathrm{f}}}\right]_{\zeta=0} \\
= & Z_{\mathrm{b}}\left(\bar{g}_{\text {lat }}, a_{\mathrm{f}} \mu\right) \frac{k}{g_{0}^{2}}\left[\frac{2}{L}-\frac{1}{a_{\mathrm{f}}} m_{2}^{\mathrm{b}}\left(a_{\mathrm{f}} / L\right) g_{0}^{2}+\mathrm{O}\left(g_{0}^{4}\right)\right] \\
= & \frac{k}{\bar{g}_{\text {lat }}^{2}(\mu)}\left[\frac{2}{L}-\frac{2}{L}\left(\gamma_{\mathrm{b}}^{(0)}+2 b_{0}\right) \log \left(a_{\mathrm{f}} \mu\right) \bar{g}_{\text {lat }}^{2}(\mu)\right. \\
& \left.-\frac{1}{a_{\mathrm{f}}} m_{2}^{\mathrm{b}}\left(a_{\mathrm{f}} / L\right) \bar{g}_{\text {lat }}^{2}(\mu)\right]+\mathrm{O}\left(\bar{g}_{\text {lat }}^{2}\right)
\end{aligned}
$$

since the computation $[48,49]$ corresponds to $\zeta=c_{\mathrm{t}}^{(1)} g_{0}^{2}+$ $\mathrm{O}\left(g_{0}^{4}\right)$. Finally, requiring finiteness of Eq. (6.23) after inserting

$\frac{1}{a_{\mathrm{f}}} m_{2}^{\mathrm{b}}\left(a_{\mathrm{f}} / L\right)=\frac{1}{L}\left[r_{2}^{\mathrm{b}}+s_{2}^{\mathrm{b}} \log \left(L / a_{\mathrm{f}}\right)+\mathrm{O}\left(a_{\mathrm{f}} / L\right)\right]$,

with [49] $r_{2}^{\mathrm{b}}=0.1683(8), \quad s_{2}^{\mathrm{b}}=0.2785(4)$ we obtain $\gamma_{\mathrm{b}}^{(0)}=s_{2}^{\mathrm{b}} / 2-2 b_{0}$ and

$\hat{\gamma}_{\mathrm{b}}=0.000(2)$. 
Note that this is the anomalous dimension of a boundary operator. Assuming that $\hat{\gamma}_{\mathrm{b}}=0$, exactly, Eq. (6.18) can now be written in the form (see also Eq. (5.14))

$\Delta K=\frac{a}{L}\left[\bar{g}^{2}\left(a^{-1}\right)\right]^{n_{\mathrm{I}}} 2 \bar{c}_{\mathrm{b}}^{\left(n_{\mathrm{I}}\right)}\left[1+\mathrm{O}\left(g^{2}\right)\right]$,

where $\bar{c}_{\mathrm{b}}^{\left(n_{I}\right)}=-c_{\mathrm{t}}^{\left(n_{\mathrm{I}}\right)}$ is the leading coefficient in

$\bar{c}_{\mathrm{b}}=\bar{c}_{\mathrm{b}}^{\left(n_{\mathrm{I}}\right)}\left[\bar{g}^{2}\left(a^{-1}\right)\right]^{n_{\mathrm{I}}}+\mathrm{O}\left(\left[\bar{g}^{2}\left(a^{-1}\right)\right]^{n_{\mathrm{I}}+1}\right)$,

as we are considering a theory where $c_{\mathrm{t}}$ is chosen to achieve $\mathrm{O}(a)$ improvement in perturbation theory, up to and including the terms $g_{0}^{2\left(n_{\mathrm{I}}-1\right)}$. The $\mathrm{O}\left(\bar{g}^{2}\left(L^{-1}\right)\right)$ term in the SymEFT matrix element is given by $r_{2}^{\mathrm{b}} \bar{g}^{2} / 2$, but it comes together with the two-loop anomalous dimension of the boundary operator and the next order correction in Eq. (6.27). Since these are presently unknown, we only show the leading order in $g^{2}$ in Eq. (6.26).

In order to compute the non-perturbative running of the coupling, one considers the step scaling function,

$\Sigma(u, a / L)=\left.\bar{g}^{2}(1 /(2 L))\right|_{\bar{g}^{2}(1 / L)=u}$,

where the choice of intermediate renormalization scheme (we chose "lat") disappears. Its leading discretization errors are (see also [43], App. A)

$$
\begin{aligned}
\Delta \Sigma(u, a / L) & =\Sigma(u, a / L)-\Sigma(u, 0) \\
& =u \frac{a}{L} \bar{c}_{\mathrm{b}}^{\left(n_{\mathrm{I}}\right)}\left[\bar{g}^{2}\left(a^{-1}\right)\right]^{n_{\mathrm{I}}}[1+\mathrm{O}(u)]
\end{aligned}
$$

Since we have seen that the one-loop anomalous dimension of $\mathcal{O}_{\mathrm{b}}$ vanishes, this is equivalent to the form used by the ALPHA collaboration recently $[43,50]$.

\section{Wilson-QCD}

Let us now briefly discuss the case of the original Wilson action for QCD including the Wilson term in the fermion action [51]. While this action is hardly used any more in the original form it is still of interest because there are results in the literature. More importantly, some large scale computations use the $\mathrm{O}(a)$-improved version with an approximate coefficient of the clover improvement term. One can gain information on the scaling of $\delta \mathcal{P}^{\mathscr{L}}$, Eq. (2.16), in that case.

The Wilson quark action breaks chiral symmetry and thus allows for the dimension five Sheikholeslami-Wohlert term [52]

$\delta \mathscr{L}^{(1)}(x)=-\omega_{\mathrm{sw}} \frac{1}{8} \bar{\psi}(x)\left[\gamma_{\mu}, \gamma_{\nu}\right] F_{\mu \nu}(x) \psi(x)$

in the SymEFT, Eq. (1.2). In principle there are additional terms proportional to quark masses, but these "only" affect quark-mass dependences [12] and are absent when one takes the continuum limit along a physical scaling trajectory defined by, for example, fixed ratios of $N_{\mathrm{f}}$ pseudo-scalar masses in the $N_{\mathrm{f}}$-flavor theory. We here neglect those $\mathrm{O}\left(a m_{\mathrm{q}}\right)$ effects; we set the quark masses to zero. There are no operators which violate rotational symmetry. Therefore, there is no mixing at $\mathrm{O}(a)$ at all. The prediction for the asymptotic $a$ dependence can then immediately be written down,

$$
\begin{aligned}
\Delta_{\mathcal{P}}(a)= & -a \bar{c}_{\mathrm{sw}}^{(0)}\left[2 b_{0} \bar{g}^{2}\left(a^{-1}\right)\right]^{\hat{\gamma}^{\mathrm{sw}}} \mathcal{M}^{\mathrm{RGI}} \\
& \times\left[1+\mathrm{O}\left(\bar{g}^{2}\left(a^{-1}\right)\right)\right] \sim a\left[\frac{1}{-\log (a \Lambda)}\right]^{\hat{\gamma}^{\mathrm{sw}}} .
\end{aligned}
$$

For the standard Wilson action, we have $\bar{c}_{\mathrm{sw}}=\bar{c}_{\mathrm{sw}}^{(0)}+$ $\mathrm{O}\left(g^{2}\right)$ with $\bar{c}_{\mathrm{sw}}^{(0)}=-1$. As in Eq. (6.30), there are additional powers of $\bar{g}^{2}\left(a^{-1}\right)$ when the theory is perturbatively $\mathrm{O}(a)$ improved [12,52-54]. We find [55] $\left(C_{\mathrm{A}}=\mathrm{N}, C_{\mathrm{F}}=\left(\mathrm{N}^{2}-\right.\right.$ $1) /(2 N))$,

$\hat{\gamma}^{\mathrm{sw}}=\frac{15 C_{\mathrm{F}}-6 C_{\mathrm{A}}}{11 C_{\mathrm{A}}-2 N_{\mathrm{f}}}$

for the anomalous dimension. It is rather small. For $\mathrm{N}=3$ this is in agreement with [33].

For the considered case of Wilson fermions, one may also easily discuss the relevant contributions from corrections to the vector and axial vector, non-singlet, flavor currents. In SymEFT, they are represented by [12]

$V_{\mu}^{r, s}(x)=\bar{\psi}_{r}(x) \gamma_{\mu} \psi_{s}(x)+a \omega_{\mathrm{V}} \partial_{\nu} T_{\mu \nu}^{r, s}(x)$,

$A_{\mu}^{r, s}(x)=\bar{\psi}_{r}(x) \gamma_{\mu} \gamma_{5} \psi_{s}(x)+a \omega_{\mathrm{A}} \partial_{\mu} P^{r, s}(x)$.

Matrix elements of interest of the corresponding lattice currents are, e.g., leptonic decay constants and semi-leptonic form factors. Using the anomalous dimensions of the nonsinglet pseudo scalar density and the tensor current [56,57], the lattice artifacts receive contributions

$$
\begin{aligned}
\Delta_{\mathcal{P}}^{\mathrm{V}}(a)= & a \bar{g}^{2}\left(a^{-1}\right)\left[2 b_{0} \bar{g}^{2}\left(a^{-1}\right)\right]^{\hat{\gamma}^{\mathrm{T}}} \\
& \times \mathcal{M}_{\mathrm{T}}^{\mathrm{RGI}}\left[\bar{c}_{\mathrm{V}}^{(1)}+\mathrm{O}\left(\bar{g}^{2}\left(a^{-1}\right)\right], \quad \hat{\gamma}^{\mathrm{T}}=\frac{3 C_{\mathrm{F}}}{11 C_{\mathrm{A}}-2 N_{\mathrm{f}}},\right. \\
\Delta_{\mathcal{P}}^{\mathrm{A}}(a)= & a \bar{g}^{2}\left(a^{-1}\right)\left[2 b_{0} \bar{g}^{2}\left(a^{-1}\right)\right]^{\hat{\gamma}^{\mathrm{P}}} \\
& \times \mathcal{M}_{\mathrm{P}}^{\mathrm{RGI}}\left[\bar{c}_{\mathrm{A}}^{(1)}+\mathrm{O}\left(\bar{g}^{2}\left(a^{-1}\right)\right], \quad \hat{\gamma}^{\mathrm{P}}=-3 \hat{\gamma}^{\mathrm{T}}, \quad(7.6)\right.
\end{aligned}
$$

where $\mathcal{M}_{\mathrm{T}}^{\mathrm{RGI}}$ is the RGI matrix element of $\partial_{\nu} T_{\mu \nu}^{r, s}$ and $\mathcal{M}_{\mathrm{P}}^{\mathrm{RGI}}$ the RGI matrix element of $\partial_{\mu} P$. There is an extra factor $\bar{g}^{2}$, as compared to previous expressions, since the $\mathrm{O}(a)$ term in the classical expansion of the currents vanishes. The $\omega_{\mathrm{V} / \mathrm{A}}^{(1)}$ factors are the one-loop matching coefficients between SymEFT and the considered lattice theory. An extended list of references with results for improvement coefficients $c_{\mathrm{V} / \mathrm{A}}^{(1)}$ for various actions is given in table 1 of [58]. The case of unimproved lattice currents, e.g. $V_{\mu, \text { latt }}^{r, s}(x)=\bar{\psi}_{r}(x) \gamma_{\mu} \psi_{s}(x)$, can be obtained by setting $\bar{c}_{\mathrm{V} / \mathrm{A}}^{(1)}=-c_{\mathrm{V} / \mathrm{A}}^{(1)}$ in Eq. (7.6). These coefficients are rather small. 


\section{Summary}

We have investigated the form of the leading discretization errors in lattice gauge theory in a few specific cases. The starting point is the leading contribution to the Symanzik effective Lagrangian in the form

$$
\begin{aligned}
& \mathscr{L}_{\mathrm{eff}}(x) \\
& =\mathscr{L}(x)+a^{n_{\min }} \sum_{i} \bar{c}_{i}^{\left(n_{\mathrm{I}}\right)} g^{2 n_{\mathrm{I}}} \mathcal{B}_{i}(x)+\cdots, \quad n_{\min } \geq 1, \quad n_{\mathrm{I}} \geq 0,
\end{aligned}
$$

where the ellipsis denotes higher powers in $g^{2}$ for each term $i$ as well as higher powers in $a$. The basis operators are chosen such that they do not mix at one-loop order and have oneloop anomalous dimensions $\gamma_{i}^{(0)} g^{2}, \gamma_{1}^{(0)} \leq \gamma_{2}^{(0)} \leq \cdots$. Once $n_{\min }, c_{i}, \gamma_{i}^{(0)}, n_{\mathrm{I}}$ are known, the leading correction to the continuum limit of spectral quantities is

$$
\begin{aligned}
\Delta_{\mathcal{P}}(a)= & a^{n_{\min }}\left[\bar{g}^{2}\left(a^{-1}\right)\right]^{n_{\mathrm{I}}}\left[2 b_{0} \bar{g}^{2}\left(a^{-1}\right)\right]^{\hat{\gamma}_{1}} \bar{c}_{1}^{\left(n_{\mathrm{I}}\right)} \\
& \times \mathcal{M}_{\mathcal{P}, 1}^{\mathrm{RGI}}\left[1+\mathrm{O}\left(\left[\bar{g}^{2}\left(a^{-1}\right)\right]^{\Delta \hat{\gamma}}, \bar{g}^{2}\left(a^{-1}\right)\right)\right] \\
& +\mathrm{O}\left(a^{n_{\min }+1}\right)
\end{aligned}
$$

with $\hat{\gamma}_{i}=\gamma_{i}^{(0)} /\left(2 b_{0}\right), \Delta \hat{\gamma}=\hat{\gamma}_{2}-\hat{\gamma}_{1}$. The only unknown is the $a$-independent renormalization group invariant matrix element $\mathcal{M}_{\mathcal{P}, 1}^{\mathrm{RGI}}$ of the operator $\mathcal{B}_{1}$. The most important ingredient in the formula is the leading $\hat{\gamma}_{1}$. In almost all considered cases, we find that $\hat{\gamma}_{1} \geq 0$ in stark contrast to the case of the $2 \mathrm{~d}$ $\mathrm{O}(3)$ model [7]. This is good news, as the leading corrections accelerate the approach to the continuum limit compared to the naive classical argumentation which neglects the overall $\left[\bar{g}^{2}\left(a^{-1}\right)\right]^{n_{\mathrm{I}}+\hat{\gamma}_{1}}$ factor.

Let us briefly summarize the results for the individual cases considered.

- Yang-Mills theory.

Discretization effects of order $a^{2}\left(n_{\min }=2\right)$ are due to two operators. Their anomalous dimensions, $\hat{\gamma}_{i}$, computed in Sect. 3, are of order one, see Eq. (3.24). In Eqs. (8.1-8.2), the original Wilson action, tree-level and one-loop Symanzik improved actions have $n_{\mathrm{I}}=0,1,2$ respectively.

- Yang-Mills theory with a boundary: Schrödinger functional.

As discussed in Sect. 6 there are linear in $a\left(n_{\min }=\right.$ 1) discretization errors due to one boundary operator. Using the literature on perturbation theory for the Schrödinger functional, we extracted its anomalous dimension and found that it vanishes within uncertainties, $\hat{\gamma}_{\mathrm{b}}=0.000(2)$. This means that the fixed order perturbation theory analysis of discretization errors carried out by the ALPHA collaboration [50] receives no logcorrections at leading order.
- Wilson $\mathrm{O}(a)$ effects due to the fermion action.

Here our analysis concerns $\mathrm{O}(a)$ effects $\left(n_{\min }=1\right)$ which come from an action with perturbative improvement, i.e. with an improvement coefficient $c_{\mathrm{sw}}$ determined at $n$-loop perturbation theory. The Pauli term, found to be the only contributing operator by Sheikholeslami and Wohlert, has $n_{\mathrm{I}}=n+1$ in Eq. (8.2). Its anomalous dimension, $\hat{\gamma}_{1}=\hat{\gamma}^{\mathrm{sw}}=\frac{15 C_{\mathrm{F}}-6 C_{\mathrm{A}}}{11 C_{\mathrm{A}}-2 N_{\mathrm{f}}}$, could be taken from the literature [33]. It is rather small. Interestingly, as one approaches the conformal window [59] by increasing $N_{\mathrm{f}}$, the anomalous dimension $\hat{\gamma}^{\text {sw }}$ grows.

- Wilson $\mathrm{O}(a)$ effects due to the flavor currents.

Weak decay (and other) matrix elements receive additional discretization errors from correction terms in the effective weak Hamiltonian. We just considered the flavor currents with perturbative $\mathrm{O}(a)$ improvement in Sect. 7. For the axial current, the (derivative of the) pseudoscalar field governs the correction term. Its $\hat{\gamma}_{\mathrm{P}}$ is negative, but relatively small in magnitude. Since the coefficient of the correction operator starts at order $g^{2}$ in perturbation theory, the total logarithmic modification, $\left[\bar{g}^{2}\left(a^{-1}\right)\right]^{n_{\mathrm{I}}}\left[2 b_{0} \bar{g}^{2}\left(a^{-1}\right)\right]^{\hat{\gamma_{\mathrm{P}}}}$, again accelerates convergence due to $n_{\mathrm{I}} \geq 1$ and $n_{\mathrm{I}}+\hat{\gamma}_{\mathrm{P}}>0$. For the vector current the $\mathrm{O}(a)$ correction involves the tensor current with $\hat{\gamma}_{\mathrm{T}}$ which is positive and rather small. This leads to an even better $a$-dependence. Note that this analysis holds also for a non-perturbatively improved action but only perturbatively improved currents.

Short distance observables $\mathcal{P}(r \Lambda)$ with $r \Lambda \ll 1$ are special. Their matrix elements $\mathcal{M}_{\mathcal{P}, i}^{\mathrm{RGI}}(r \Lambda)$ are computable in renormalized perturbation theory in terms of the coupling at scale $\mu=1 / r$ and one can make parameter free predictions for the leading corrections. As discussed in Sect. 5.2 the usual tree-level improved observables do not always lead to a reduction of the asymptotic cutoff effects, but this is easy to fix so as to have cutoff-effects suppressed by one power of $\bar{g}^{2}\left(r^{-1}\right)$ at short distances.

As a general conclusion, our results are very positive because the so-far known logarithmic corrections are relatively small. This lends support to some of the continuum extrapolations performed in the literature. For example, the BMW collaboration has performed continuum extrapolations of data obtained with tree-level coefficient, $c_{\mathrm{sw}}=1$ of the Sheikholeslami-Wohlert term [60]. In principle, the asymptotic behavior is then $\bar{c}_{\mathrm{sw}}^{(1)} \bar{g}^{2}\left(a^{-1}\right)\left[2 b_{0} \bar{g}^{2}\left(a^{-1}\right)\right]^{\hat{\gamma}^{\mathrm{sw}}}$. In one of their continuum extrapolations they used this form but with $\hat{\gamma}^{\text {sw }} \rightarrow 0$, which we now see is a rather good approximation. Of course, the difficult question in such extrapolations is whether one is in the region where the asymptotics dominates. For this reason they also used alternative extrapolation functions. 
Despite the small values of $\hat{\gamma}$ that we found, with treelevel or one-loop Symanzik improved action, the $\left[\bar{g}^{2}\left(a^{-1}\right)\right]^{n_{\mathrm{I}}}$ $\left[2 b_{0} \bar{g}^{2}\left(a^{-1}\right)\right]^{\hat{\gamma}_{1}}$ effects are non-negligible when MC results are accurate, see the right part of Fig. 3. In any case, when the leading behavior is known, it should be incorporated into the fit function. Still, we emphasize that the asymptotically leading behavior can be predicted, not the region where exactly this dominates over formally suppressed terms.

Of course the most interesting application of SymEFT is lattice QCD with $n_{\min }=2$ in Eq. (1.3). In that case the basis of contributing operators is considerably larger. Work on determining their anomalous dimensions is in progress [55]. Also Gradient flow observables are of high interest. Their discretization errors are surprisingly large [61-63]. Now that it is known that standard pure gauge theory operators are not the source of this behavior, since they have positive $\hat{\gamma}_{i}$, a natural suspicion is that there is an unusually large and negative anomalous dimension $\hat{\gamma}$ of the additional dimension six operator at $t \rightarrow 0$, present in the 5-d formulation of the Gradient Flow, see [64] for more details. We also plan to investigate this issue.

Acknowledgements We thank Hubert Simma and Kay Schönwald for many discussions and Jean Zinn-Justin for communication on irrelevant operators and the $\epsilon$-expansion. We acknowledge funding by the $\mathrm{H} 2020$ program in the Europlex training network, grant agreement No. 813942.

Note added in proof The anomalous dimension of the boundary operator discussed in Sect. 6 vanishes also beyond one-loop as can be argued as follows. Differentiating expectation values with respect to $T$ in the continuum formulation can be written as an insertion of the Hamiltonian at the boundary $x_{0}=T$ (see also Appendix D of Ref. [12]).

Due to the assumed boundary condition we have $F_{k l}(T, \mathbf{x})=0$ and the (integrated) boundary field equals the Hamiltonian, which has no anomalous dimension. We thank M. Lüscher for suggesting to differentiate with respect to $T$ to prove the vanishing of the anomalous dimension.

Data Availability Statement This manuscript has no associated data or the data will not be deposited. [Authors' comment: All necessary details are given in the manuscript.]

Open Access This article is licensed under a Creative Commons Attribution 4.0 International License, which permits use, sharing, adaptation, distribution and reproduction in any medium or format, as long as you give appropriate credit to the original author(s) and the source, provide a link to the Creative Commons licence, and indicate if changes were made. The images or other third party material in this article are included in the article's Creative Commons licence, unless indicated otherwise in a credit line to the material. If material is not included in the article's Creative Commons licence and your intended use is not permitted by statutory regulation or exceeds the permitted use, you will need to obtain permission directly from the copyright holder. To view a copy of this licence, visit http://creativecomm ons.org/licenses/by/4.0/.

Funded by SCOAP ${ }^{3}$.

\section{References}

1. K. Symanzik, Cutoff dependence in lattice $\phi_{4}^{4}$ theory. NATO Sci. Ser. B 59, 313 (1980). https://doi.org/10.1007/ 978-1-4684-7571-5_18

2. K. Symanzik, Some Topics in Quantum Field Theory, in Mathematical Problems in Theoretical Physics. Proceedings, 6th International Conference on Mathematical Physics, West Berlin, Germany, August 11-20, 1981, pp. 47-58, (1981)

3. K. Symanzik, Continuum limit and improved action in lattice theories. 1. Principles and phi**4 theory. Nucl. Phys. B 226, 187 (1983). https://doi.org/10.1016/0550-3213(83)90468-6

4. K. Symanzik, Continuum limit and improved action in lattice theories. 2. $\mathrm{O}(\mathrm{N})$ Nonlinear sigma model in perturbation theory. Nucl. Phys. B 226, 205 (1983). https://doi.org/10.1016/ 0550-3213(83)90469-8

5. P. Weisz, Renormalization and lattice artifacts, in Modern perspectives in lattice QCD: quantum field theory and high performance computing. Proceedings, International School, 93rd Session, Les Houches, France, August 3-28, 2009, pp. 93-160, (2010), arXiv: 1004.3462

6. J. Zinn-Justin, Quantum field theory and critical phenomena. Int. Ser. Monogr. Phys. 113, (2002). Chapter 27

7. J. Balog, F. Niedermayer, P. Weisz, Logarithmic corrections to $\mathrm{O}\left(a^{2}\right)$ lattice artifacts. Phys. Lett. B 676, 188 (2009). https://doi. org/10.1016/j.physletb.2009.04.082. arXiv:0901.4033

8. J. Balog, F. Niedermayer, P. Weisz, The puzzle of apparent linear lattice artifacts in the $2 \mathrm{~d}$ non-linear sigma-model and 's solution. Nucl. Phys. B 824, 563 (2010). https://doi.org/10.1016/j. nuclphysb.2009.09.007. arXiv:0905.1730

9. M. Lüscher, P. Weisz, U. Wolff, A Numerical method to compute the running coupling in asymptotically free theories. Nucl. Phys. B 359, 221 (1991). https://doi.org/10.1016/0550-3213(91)90298-C

10. F. Knechtli, B. Leder, U. Wolff, Cutoff effects in $\mathrm{O}(\mathrm{N})$ nonlinear sigma models. Nucl. Phys. B 726, 421 (2005). https://doi.org/10. 1016/j.nuclphysb.2005.08.002. arXiv:hep-lat/0506010

11. G. Heatlie, G. Martinelli, C. Pittori, G.C. Rossi, C.T. Sachrajda, The improvement of hadronic matrix elements in lattice QCD. Nucl. Phys. B 352, 266 (1991). https://doi.org/10.1016/ 0550-3213(91)90137-M

12. M. Lüscher, S. Sint, R. Sommer, P. Weisz, Chiral symmetry and $\mathrm{O}$ (a) improvement in lattice QCD. Nucl. Phys. B 478, 365 (1996). https://doi.org/10.1016/0550-3213(96)00378-1. arXiv:hep-lat/9605038

13. R. Narayanan, H. Neuberger, Infinite $\mathrm{N}$ phase transitions in continuum Wilson loop operators. JHEP 0603, 064 (2006). https://doi. org/10.1088/1126-6708/2006/03/064

14. M. Lüscher, Properties and uses of the Wilson flow in lattice QCD. JHEP 1008, 071 (2010). https://doi.org/10.1007/ JHEP08(2010)071

15. P. Weisz, Continuum limit improved lattice action for pure YangMills theory. 1. Nucl. Phys. 212, 1 (1983). https://doi.org/10.1016/ 0550-3213(83)90595-3

16. M. Lüscher, P. Weisz, On-shell improved lattice gauge theories. Comm. Math. Phys. 97, 59 (1985)

17. R. Alonso, E.E. Jenkins, A.V. Manohar, M. Trott, Renormalization group evolution of the standard model dimension six operators III: gauge coupling dependence and phenomenology. JHEP 04, 159 (2014). https://doi.org/10.1007/JHEP04(2014)159. arXiv: 1312.2014

18. R. Sommer, Introduction to Non-perturbative Heavy Quark Effective Theory, in Modern perspectives in lattice QCD: quantum field theory and high performance computing. Proceedings, International School, 93rd Session, Les Houches, France, August 3-28, 2009, pp. 517-590, (2010), arXiv:1008.0710 
19. J.A. Gracey, Classification and one loop renormalization of dimension-six and dimension-eight operators in quantum gluodynamics. Nucl. Phys. B 634, 192 (2002). https://doi.org/10.1016/ S0550-3213(02)00334-6. arXiv:hep-ph/0204266

20. S.D. Joglekar, B.W. Lee, General theory of renormalization of gauge invariant operators. Ann. Phys. 97, 160 (1976). https://doi. org/10.1016/0003-4916(76)90225-6

21. J.C. Collins, R.J. Scalise, The renormalization of composite operators in Yang-Mills theories using general covariant gauge. Phys. Rev. D 50, 4117 (1994). https://doi.org/10.1103/PhysRevD.50. 4117. arXiv:hep-ph/9403231

22. B.S. DeWitt, Quantum theory of gravity. 2. The manifestly covariant theory. Phys. Rev. 162, 1195 (1967). https://doi.org/10.1103/ PhysRev.162.1195

23. H. Kluberg-Stern, J.B. Zuber, Renormalization of nonabelian gauge theories in a background field gauge. 1. Green functions. Phys. Rev. D 12, 482 (1975). https://doi.org/10.1103/PhysRevD. 12.482

24. L.F. Abbott, The background field method beyond one loop. Nucl. Phys. B 185, 189 (1981). https://doi.org/10.1016/ 0550-3213(81)90371-0

25. M. Lüscher, P. Weisz, Background field technique and renormalization in lattice gauge theory. Nucl. Phys. B 452, 213 (1995). https:// doi.org/10.1016/0550-3213(95)00346-T. arXiv:hep-lat/9504006

26. L. Faddeev, V. Popov, Feynman diagrams for the Yang-Mills field. Phys. Lett. B 25, 29 (1967). https://doi.org/10.1016/ 0370-2693(67)90067-6

27. M. Misiak, M. Münz, Two loop mixing of dimension five flavor changing operators. Phys. Lett. B 344, 308 (1995). https://doi.org/ 10.1016/0370-2693(94)01553-O. arXiv:hep-ph/9409454

28. K.G. Chetyrkin, M. Misiak, M. Münz, Beta functions and anomalous dimensions up to three loops. Nucl. Phys. B 518, 473 (1998). https://doi.org/10.1016/S0550-3213(98)00122-9. arXiv:hep-ph/9711266

29. T. Luthe, A. Maier, P. Marquard, Y. Schröder, Complete renormalization of QCD at five loops. JHEP 03, 020 (2017). https://doi.org/ 10.1007/JHEP03(2017)020. arXiv:1701.07068

30. P. Nogueira, Automatic feynman graph generation. J. Comput. Phys. 105, 279 (1993). https://doi.org/10.1006/jcph.1993.1074

31. P. Nogueira, Abusing qgraf. Nucl. Instrum. Meth. A559, 220 (2006). https://doi.org/10.1016/j.nima.2005.11.151

32. J.A.M. Vermaseren, New features of FORM, arXiv:math-ph/0010025

33. S. Narison, R. Tarrach, Higher dimensional renormalization group invariant vacuum condensates in quantum chromodynamics. Phys. Lett. B 125, 217 (1983). https://doi.org/10.1016/ 0370-2693(83)91271-6

34. Y. Iwasaki, Renormalization Group Analysis of Lattice Theories and Improved Lattice Action. II. Four-dimensional non-Abelian SU(N) gauge model, arXiv:1111.7054

35. QCD-TARO collaboration, P. de Forcrand et al., Search for effective lattice action of pure QCD, Nucl. Phys. Proc. Suppl. 53 (1997) 938. https://doi.org/10.1016/S0920-5632(96)00820-1. arXiv:hep-lat/9608094

36. T. Takaishi, Heavy quark potential and effective actions on blocked configurations. Phys. Rev. D 54, 1050 (1996). https://doi.org/10. 1103/PhysRevD.54.1050

37. R. Sommer, A New way to set the energy scale in lattice gauge theories and its applications to the static force and alpha-s in SU(2) Yang-Mills theory. Nucl. Phys. B 411, 839 (1994). https://doi.org/ 10.1016/0550-3213(94)90473-1. arXiv:hep-lat/9310022

38. K. Symanzik, Concerning the continuum limit in some lattice theories. J. Phys. Colloq. 43, 254 (1982). https://doi.org/10.1051/ jphyscol: 1982350

39. S. Necco, R. Sommer, The $\mathrm{N}(\mathrm{f})=0$ heavy quark potential from short to intermediate distances. Nucl. Phys. B 622,
328 (2002). https://doi.org/10.1016/S0550-3213(01)00582-X. arXiv:hep-lat/0108008

40. AlPHA collaboration, G. de Divitiis, R. Frezzotti, M. Guagnelli, M. Lüscher, R. Petronzio, R. Sommer et al., Universality and the approach to the continuum limit in lattice gauge theory, Nucl. Phys. B437 (1995) 447. https://doi.org/10.1016/0550-3213(94)00019-B arXiv:hep-lat/9411017

41. ALPHA collaboration, A. Bode, P. Weisz, U. Wolff, Two loop computation of the Schrodinger functional in lattice QCD, Nucl. Phys. B576 (2000) 517. https://doi.org/10.1016/ S0550-3213(00)00187-5, arXiv:hep-lat/9911018

42. ETM collaboration, C. Alexandrou, M. Constantinou, H. Panagopoulos, Renormalization functions for $\mathrm{Nf}=2$ and $\mathrm{Nf}=4$ twisted mass fermions, Phys. Rev. D95 (2017) 034505. https://doi. org/10.1103/PhysRevD.95.034505 arXiv:1509.00213

43. ALPHA collaboration, M. Dalla Brida, P. Fritzsch, T. Korzec, A. Ramos, S. Sint, R. Sommer, A non-perturbative exploration of the high energy regime in $N_{\mathrm{f}}=3$ QCD, Eur. Phys. J. C78 (2018) 372. https://doi.org/10.1140/epjc/s10052-018-5838-5 arXiv: 1803.10230

44. S. Necco, R. Sommer, Testing perturbation theory on the $\mathrm{N}(\mathrm{f})=0$ static quark potential. Phys. Lett. B 523, 135 (2001). https://doi. org/10.1016/S0370-2693(01)01298-9. arXiv:hep-ph/0109093

45. M. Lüscher, R. Narayanan, P. Weisz, U. Wolff, The Schrödinger functional: a renormalizable probe for non-abelian gauge theories. Nucl. Phys. B 384, 168 (1992). https://doi.org/10.1016/ 0550-3213(92)90466-O. arXiv:hep-lat/9207009

46. M. Lüscher, R. Sommer, P. Weisz, U. Wolff, A Precise determination of the running coupling in the SU(3) Yang-Mills theory. Nucl. Phys. B 413, 481 (1994). https://doi.org/10.1016/ 0550-3213(94)90629-7. arXiv:hep-lat/9309005

47. P. Perez-Rubio, S. Sint, S. Takeda, An O(a) modified lattice set-up of the Schródinger functional in SU(3) gauge theory. JHEP 07, 116 (2011). https://doi.org/10.1007/JHEP07(2011)116. arXiv: 1105.0110

48. ALPHA collaboration, A. Bode, U. Wolff, P. Weisz, Two loop computation of the Schrodinger functional in pure SU(3) lattice gauge theory, Nucl. Phys. B540 (1999) 491. https://doi.org/10. 1016/S0550-3213(98)00772-X arXiv:hep-lat/9809175

49. A. Bode, "PhD thesis, Humboldt University, August 1998, http:// dochost.rz.hu-berlin.de/dissertationen/physik/ (in German)."

50. ALPHA collaboration, M. Bruno, M. Dalla Brida, P. Fritzsch, T. Korzec, A. Ramos, S. Schaefer et al., QCD Coupling from a Nonperturbative Determination of the Three-Flavor $\Lambda$ Parameter, Phys. Rev. Lett. 119 (2017) 102001. https://doi.org/10.1103/ PhysRevLett.119.102001 arXiv:1706.03821

51. K.G. Wilson, Confinement of quarks. Phys. Rev. D 10, 2445 (1974). https://doi.org/10.1103/PhysRevD.10.2445

52. B. Sheikholeslami, R. Wohlert, Improved continuum limit lattice action for QCD with wilson fermions. Nucl. Phys. B 259, 572 (1985). https://doi.org/10.1016/0550-3213(85)90002-1

53. R. Wohlert, Improved continuum limit lattice action for quarks, DESY-87-069 (1987)

54. S. Aoki, Y. Kuramashi, Determination of the improvement coefficient $\mathrm{c}(\mathrm{SW})$ up to one loop order with the conventional perturbation theory. Phys. Rev. D 68, 094019 (2003). https://doi.org/10.1103/ PhysRevD.68.094019. arXiv:hep-lat/0306015

55. N. Husung, P. Marquard, R. Sommer, in preparation

56. S.A. Larin, The renormalization of the axial anomaly in dimensional regularization. Phys. Lett. B 303, 113 (1993). https://doi. org/10.1016/0370-2693(93)90053-K. arXiv:hep-ph/9302240

57. D.J. Broadhurst, A.G. Grozin, Matching QCD and HQET heavy - light currents at two loops and beyond. Phys. Rev. D 52, 4082 (1995). https://doi.org/10.1103/PhysRevD.52.4082. arXiv:hep-ph/9410240 
58. R. Sommer, Non-perturbative QCD: Renormalization, $\mathrm{O}(\mathrm{a})$ improvement and matching to Heavy Quark Effective Theory, in Workshop on Perspectives in Lattice QCD Nara, Japan, October 31-November 11, 2005, (2006), arXiv:hep-lat/0611020

59. D. Nogradi, A. Patella, Strong dynamics, composite Higgs and the conformal window. Int. J. Mod. Phys. A 31, 1643003 (2016). https://doi.org/10.1142/S0217751X1643003X. arXiv:1607.07638

60. S. Dürr, Z. Fodor, C. Hoelbling, S.D. Katz, S. Krieg, T. Kurth et al., Lattice QCD at the physical point: light quark masses. Phys. Lett. B 701, 265 (2011). https://doi.org/10.1016/j.physletb.2011. 05.053. arXiv: 1011.2403

61. R. Sommer, Scale setting in lattice QCD. PoS LATTICE2013, 1518 (2014). https://doi.org/10.22323/1.187.0015. arXiv:1401.3270
62. S. Sint, A. Ramos, On $\mathrm{O}\left(a^{2}\right)$ effects in gradient flow observables. PoS LATTICE2014, 329 (2015). https://doi.org/10.22323/1.214. 0329. arXiv: 1411.6706

63. ALPHA collaboration, M. Dalla Brida, P. Fritzsch, T. Korzec, A. Ramos, S. Sint, R. Sommer, Slow running of the Gradient Flow coupling from $200 \mathrm{MeV}$ to $4 \mathrm{GeV}$ in $N_{\mathrm{f}}=3 \mathrm{QCD}$, Phys. Rev. D95 (2017) 014507. https://doi.org/10.1103/PhysRevD.95. 014507 arXiv: 1607.06423

64. A. Ramos, S. Sint, Symanzik improvement of the gradient flow in lattice gauge theories. Eur. Phys. J. C 76, 15 (2016). https://doi. org/10.1140/epjc/s10052-015-3831-9. arXiv:1508.05552 